\title{
ON $K_{3}$ OF TRUNCATED POLYNOMIAL RINGS
}

\author{
BY
}

JANET AISBETT

\begin{abstract}
Group homology spectral sequences are used to investigate $K_{3}$ of truncated polynomial rings. If $F$ is a finite field of odd characteristic, we show that relative $K_{2}$ of the pair $\left(F[t] /\left(t^{q}\right),\left(t^{k}\right)\right)$, which has been identified by van der Kallen and Stienstra, is isomorphic to $K_{3}\left(F[t] /\left(t^{k}\right),(t)\right)$ when $q$ is sufficiently large. We also show that $H_{3}\left(\mathrm{SL} \mathbf{Z}[t] /\left(t^{k}\right) ; \mathbf{Z}\right)=\mathbf{Z}^{k-1} \oplus \mathbf{Z} / 24$ and is isomorphic to the associated $K_{3}$ group modulo an elementary abelian 2-group.
\end{abstract}

1. Introduction. There are relatively few rings whose third algebraic $K$-groups are known fully. Group homology spectral sequence techniques, however, have proved a useful tool, yielding for example $K_{3}$ of the quotient rings of the rational integers [ALSS]. This paper again applies these methods. Our main results are for $k \geqslant 2$ :

1.1 THEOREM. There are exact sequences

$$
\begin{aligned}
\mathbf{Z} \oplus \mathbf{Z} \oplus \mathbf{Z} / 2 \stackrel{1 \oplus 1 \oplus \iota}{\rightarrow} K_{3} \mathbf{Z}[t] /\left(t^{k+1}\right) \\
\quad=K_{3} \mathbf{Z} \oplus \mathbf{Z}^{k} \oplus U_{k+1} \rightarrow K_{3} \mathbf{Z}[t] /\left(t^{k}\right) \rightarrow \mathbf{Z},
\end{aligned}
$$

where $U_{k+1}$ is an elementary abelian 2-group of rank at most $[(k+1) / 2]$, and $\iota=0$ if $k$ is even.

1.2 THEOREM. If $F$ is a finite field of odd characteristic, there is an isomorphism

$$
\partial^{-1} \circ \Delta_{k}: K_{1}\left(F[t] /\left(t^{2 k}\right),(t)\right) /\left\{1-\alpha t^{k}: \alpha \in F\right\} \rightarrow K_{3}\left(F[t] /\left(t^{k}\right),(t)\right) \text {. }
$$

These theorems use, and sharpen, results of Van der Kallen and Stienstra [S, VKS]. Stienstra obtains 1.1 modulo information on the torsion group $U_{k}$; the theorem with $k=2$ has essentially been proved by Kassel. Van der Kallen and Stienstra define the isomorphism $\Delta_{k}$ onto the relative $K$-group $K_{2}\left(F[t] /\left(t^{q}\right),\left(t^{k}\right)\right)$, whenever $q \gg k$ and $F$ is a perfect field. Theorem 1.2 with $k=2$ and 3 has been proved by Snaith, Lluis and Aisbett [LS, ALS].

The Hochschild-Serre spectral sequences studied are associated to the reduction $\Pi^{k}: \mathrm{SL}_{n} R[t] /\left(t^{k}\right) \rightarrow \mathrm{SL}_{n} R$, where $R$ is initially any commutative ring with identity for which $S K_{1} R=0$. More general results than those of Theorems 1.1 and 1.2 would need information on certain $E_{* *}^{2}$ terms.

Received by the editors March 20, 1984 and, in revised form, February 19, 1985.

1980 Mathematics Subject Classification. Primary 18F25, 20G10, 20J06; Secondary 18G40, 18 G35. 
The remainder of this paper is organized as follows. $\$ 2$ lists notation, and introduces Hochschild-Serre spectral sequences from our perspective. $\$ 3$ recursively estimates the second homology groups of the kernel of $\Pi^{k}$, by means of spectral sequences associated to the reduction $\operatorname{ker} \Pi^{k} \rightarrow \operatorname{ker} \Pi^{k-1}$; this also gives information on the $\mathrm{SL}_{n} R$-coinvariance of the third homology groups. $\S 4$ deals with the integral case, to prove 1.1 , and $\$ 5$ deals with the finite fields to prove 1.2 . The appendix contains constructive proofs of various module structural details needed elsewhere in the paper.

ACKNOWLEDGEMENT. I am indebted to an unknown referee for his extremely detailed and patient comments on an earlier version of this paper.

2. Notation and conventions. This section introduces notation, describes differentials in Hochschild-Serre spectral sequences, and determines a formula for the $d^{2}$-differential which will cover our applications. There are five subsections.

\subsection{General notation and conventions.}

2.1.1 $R$ is a commutative associative ring with identity, such that for some $N>4$ and all $n \geqslant N, S K_{1}(n, R)=0$ (i.e. the elementary matrices generate $\mathrm{SL}_{n} R$ ) and $K_{2}(n, R)=K_{2} R$. Henceforth assume that $n \geqslant N$.

2.1.2 $R_{k}$ is the truncated polynomial ring $R[t] /\left(t^{k}\right)$.

2.1.3 Any map $\pi$ is a homomorphism induced by the reduction $R_{k} \rightarrow R_{r}$ for some $r<k$ which the context will indicate. $\pi^{k}$ is induced by the reduction $R_{k} \rightarrow R_{k-1}$.

2.1.4 All diagrams are commutative exact and all sequences are exact unless otherwise specified.

2.2 Notation for elements and $\mathrm{SL}_{n} R$-submodules of $\mathrm{SL}_{n} R_{k}$.

2.2.1 SL $R \equiv \mathrm{SL}_{\infty} R \equiv \lim _{n} \mathrm{SL}_{n} R$, where the special linear group $\mathrm{SL}_{n} R$ includes into $\mathrm{SL}_{n+1} R$ as the upper left corner matrices, say. An elementary matrix is denoted $e_{i j}(\alpha), \quad i \neq j, \quad \alpha \in R$. Set $\ddot{e}_{i j}(\alpha)=\operatorname{diag}\left(1,1, \ldots, 1+\alpha, \ldots,(1+\alpha)^{-1}, \ldots 1\right)$ when $(1+\alpha)$ is invertible in $R$; here, the nontrivial entries are in the $i$ th and $j$ th positions.

2.2.2 $\underline{\alpha}_{i j}$ is the $n \times n$ matrix over $R$ with $\alpha \in R$ in the $(i, j)$ th position and all other entries zero; $\underline{\alpha}_{i j}=\underline{\alpha}_{i i}-\underline{\alpha}_{j j} . M_{n}^{0}$ is the $\mathrm{SL}_{n} R$-module of zero-trace $n \times n$ matrices over $R$. (Here and elsewhere, subscript ranges are implied to be $\{1, \ldots, n\}$.)

2.2.3 $G_{n}^{k}=\operatorname{ker}\left(\pi_{*}: \mathrm{SL}_{n} R_{k} \rightarrow \mathrm{SL}_{n} R\right)$ so that $\mathrm{SL}_{n} R_{k}=\mathrm{SL}_{n} R \ltimes G_{n}^{k}$. Let $i: G_{n}^{k} \rightarrow$ $\mathrm{SL}_{n} R_{k}$ be the inclusion. The kernel of $\pi_{*}: G_{n}^{k+1} \rightarrow G_{n}^{k}$ is central in $G_{n}^{k+1}$ and is isomorphic to $M_{n}^{0}$ (identify $e_{i j}\left(\alpha t^{k}\right)$ with $\left.\underline{\alpha}_{i j}\right)$. We denote it $M_{n}^{0}\left(t^{k}\right)$, or sometimes just $M_{n}^{0}$. Let $j: M_{n}^{0}\left(t^{k}\right) \rightarrow G_{n}^{k+1}$ be the inclusion.

2.3 Commutator relations in $\mathrm{SL}_{n} R_{k}$ : the $\mathrm{SL}_{n} R$-action on $M_{n}^{0}$. If $x, y \in R_{k}$ and $x^{2} y^{2}=0$,

$$
\left[e_{i j}(x), e_{a b}(y)\right]=\left\{\begin{array}{l}
e_{i b}(x y), \quad j=a, i \neq b \\
e_{a j}(-x y), \quad i=b, j \neq a, \\
\ddot{e}_{i j}(x y) e_{i j}\left(-x^{2} y\right) e_{j i}\left(x y^{2}\right), \quad i=b, j=a, \\
0, \text { else. }
\end{array}\right.
$$


If $\alpha, \beta \in R$, the (left) $\mathrm{SL}_{n} R$-action on $M_{n}^{0}$ is

$$
\left(e_{i j}(\alpha)-1\right) \cdot \underline{\beta}_{a b}=\left\{\begin{array}{l}
\underline{\alpha \beta}_{i b}, \quad j=a, i \neq b, \\
-\frac{\alpha \beta}{a j}, \quad a \neq j, i=b, \\
\frac{\alpha \underline{\beta}}{i j}_{0,} \text { otherwise. }
\end{array}\right.
$$

(The right action differs only by the sign reversal $\alpha \rightarrow-\alpha$.)

2.4 Homology related definitions.

2.4.1 $H_{*} X$ denotes the integral homology group $H_{*}(X ; \mathbf{Z})$ of a group $X$.

2.4.2 $H_{1}\left(\mathrm{SL}_{n} R ; M_{n}^{0}\right)=H H_{1}(R, R)$, where $H H$ denotes Hochschild homology [K4, 2.16]. Since $R$ is commutative,

$$
H H_{1}(R, R)=R \otimes R /\langle\alpha \otimes \beta \gamma-\alpha \beta \otimes \gamma+\beta \otimes \gamma \alpha: \alpha, \beta, \gamma \in R\rangle
$$

(e.g. [I, p. 108]) and is isomorphic to the $R$-module of absolute Kähler differentials $\Omega \equiv \Omega_{R / \mathbf{z}}^{1}$ (e.g. [K3]).

2.4.3 $B_{*} X$ is the standard normalized bar resolution with $\mathbf{Z}$-basis elements $x_{0}\left[x_{1}|\cdots| x_{n}\right], x_{i} \in X$. The boundary map $\partial_{X}: B_{*} X \rightarrow B_{*-1} X$ is

$$
\begin{aligned}
\partial_{X} x_{0}\left[x_{1}|\cdots| x_{n}\right]= & x_{0} x_{1}\left[x_{2}|\cdots| x_{n}\right]-\left(\sum_{i=2}^{n}(-1)^{i} x_{0}\left[x_{1}|\cdots| x_{i-1} x_{i} \mid \cdots x_{n}\right]\right) \\
& +(-1)^{n} x_{0}\left[x_{1}\left|x_{2} \cdots\right| x_{n-1}\right] .
\end{aligned}
$$

2.4.4 If $C$ is a right $\mathbf{Z}[X]$ coefficient module, and $x \in C \otimes_{X} B_{*} X$, we write $\{x\}$ for the class of $x$ with respect to the equivalence relation induced by the boundary $\operatorname{map} 1 \otimes \partial_{X}$.

2.4.5 $[x \cap y]$ denotes $[x \mid y]-[y \mid x] \in B_{2} X$; similarly, $\left[x_{1} \cap x_{2} \cap \cdots \cap x_{r-1} \cap x_{r}\right]$ is $\Sigma(-1)^{\sigma}\left[\sigma x_{1}\left|\sigma x_{2}\right| \cdots \mid \sigma x_{r}\right]$, where the sum if over the symmetric group $\sum_{r}$. Generally, if $z=z_{0}\left[z_{1} \mid \cdots z_{i}\right] \in B_{i} X$ and $z^{1}=z_{0}^{1}\left[z_{i+1} \mid \cdots z_{i+j}\right] \in B_{j} X$, then $\left[z \cap z^{1}\right] \in$ $B_{i+j} X$ is

$$
\begin{array}{r}
\sum\left\{(-1)^{\sigma} z_{0} z_{0}^{1}\left[z_{\sigma(1)}|\cdots| z_{\sigma(i+j)}\right]: \sigma \in \sum_{i+j} ; \sigma^{-1}(1)<\sigma^{-1}(2)<\cdots<\sigma^{-1}(i)\right. \\
\text { and } \left.\sigma^{-1}(i+1)<\sigma^{-1}(i+2)<\cdots<\sigma^{-1}(i+j)\right\} .
\end{array}
$$

(That is, $\sigma$ runs over all $(i, j)$ - shuffles-see [M, p. 243].) Extend [- $\cap-]$ to a group homomorphism $B_{*} X \otimes B_{*} X \rightarrow B_{*} X$.

2.4.6 If $X$ is abelian with operation $+, \cap: H_{i} X \otimes H_{j} X \rightarrow H_{i+j} X \times X \stackrel{(+)_{*}}{\rightarrow} H_{i+j} X$ is the homology (shuffle) product. This operation defines an exterior algebra $\Lambda^{*} X$ which injects into $H_{*} X$ (see [B, p. 123]).

2.4.7 Suppose $X_{1}$ and $X_{2}$ are subgroups of $X$ with $\left[X_{1}, X_{2}\right]=1$. Identify $x \in B_{i} X_{1}$ and $x^{\prime} \in B_{j} X_{2}$ with their images in $B_{*} X$. Then

$$
\partial_{X}\left[x \cap x^{\prime}\right]=\left[\partial_{X}(x) \cap x^{\prime}\right]+(-1)^{i}\left[x \cap \partial_{X}\left(x^{\prime}\right)\right] .
$$

Note that this is not true for general elements in $B_{*} X$. 
2.5 Hochschild-Serre spectral sequences. This subsection reviews aspects of Hochschild-Serre group homology spectral sequences and it introduces more notation $[\mathbf{C E}]$.

2.5.1 Take an extension $N \stackrel{i}{\rightarrow} Y \stackrel{f}{\rightarrow} X$, in a category of $S$-groups with $S$-equivariant maps, say. Identify $N$ with $i(N)$. Let $C$ be a right $Y$-module. Consider the spectral sequence

$$
E_{* *}^{2}(C)=H_{*}\left(X ; H_{*}(N ; C)\right) \Rightarrow H_{*}(Y ; C)
$$

induced from the bicomplex $\left\{C \otimes_{Y}\left(B_{*} Y \otimes B_{*} X\right) \equiv\left(C \otimes_{N} B_{*} Y\right) \otimes_{X} B_{*} X ; \partial=d_{\mathrm{I}}\right.$ $\left.+d_{\mathrm{II}}\right\} . B_{*} X$ is the standard bar resolution, with $d_{\mathrm{II}}=\partial_{Y} \otimes 1$ and $d_{\mathrm{I}}(f \otimes g)=$ $(-1)^{|f|} f \otimes \partial_{X} g . B_{*} Y \otimes B_{*} X$ is a left $Y$-module via diagonal action, and $C \otimes_{N} B_{*} Y$ is a right $X$-module with action $c \otimes y \cdot x=(c \cdot \hat{x}) \otimes\left(\hat{x}^{-1} y\right)(\hat{x}$ is any lifting of $x$ to $Y)$. This is well defined, since for all $n$ in $N, c \cdot \hat{x} \otimes \hat{x}^{-1} y=c \cdot \hat{x} n \otimes n^{-1} \hat{x}^{-1} y$.

2.5.2 If $x \in E_{a, b}^{c}(C)$ and $2 \leqslant c \leqslant a$, then $d_{a, b}^{c} x$ is calculated by choosing representatives $x_{i} \in C \otimes_{Y}\left(B_{b+i} Y \otimes B_{a-i} X\right)$ for $0 \leqslant i<c$, such that $x_{0}$ represents $x$ and $d_{\mathrm{I}} x_{i}=-d_{\mathrm{II}} x_{i+1}$; set $d_{a, b}^{c} x=\left\{d_{\mathrm{I}} x_{c-1}\right\}$. Let $d_{a, b}^{c} x_{0}$ denote $d_{\mathrm{I}} x_{c-1}$ (of course, this definition is not unique).

2.5.3 Suppose $C$ is a trivial $Y$-module. Suppose $Y_{1}$ and $Y_{2}$ are subgroups of $Y$ with $\left[Y_{i}, N\right]=1, i=1,2$, and, if $f\left(Y_{i}\right)=X_{i},\left[X_{1}, X_{2}\right]=1$. Let $u: X \rightarrow Y$ be any set map section to $f$ with $u\left(X_{i}\right) \subset Y_{i}$. Suppose

$$
x=\sum_{i \in I}(-1)^{i}\left[x_{1}^{i}\left|x_{2}^{i}\right| \cdots \mid x_{a}^{i}\right] \in B_{a} X_{1}
$$

and

$$
v=\sum_{j \in J}(-1)^{j}\left[v_{1}^{j}\left|v_{2}^{j}\right| \cdots \mid v_{b}^{j}\right] \in B_{b} X_{2}
$$

represent elements in $H_{*} X$ and $y \in C \otimes_{N} B_{s} N$ is a cycle. Then in the spectral sequence (2.4),

$$
\begin{aligned}
d_{a+b, s}^{2}\{y \otimes[x \cap v]\}= & \left\{\left[d_{a, s}^{2}(y \otimes x) \cap v\right]\right\}+(-1)^{a b}\left\{\left[d_{b, s}^{2}(y \otimes v) \cap x\right]\right\} \\
& +\left\{\sum_{i \in I} \sum_{j \in J}(-1)^{a+i+j}\left[\left[\left[u\left(x_{1}^{i}\right)^{-1}, u\left(v_{1}^{j}\right)^{-1}\right]\right] \cap y\right]\right. \\
& \left.\otimes\left[\left[x_{2}^{i}|\cdots| x_{a}^{i}\right] \cap\left[v_{2}^{j}|\cdots| v_{b}^{j}\right]\right]\right\} .
\end{aligned}
$$

Note. (1) In the process of calculating the differential we prove that $y \otimes[x \cap v]$ represents an element in $H_{a+b}\left(X ; H_{s}(N ; C)\right)$.

(2) The restricted definitions of $x, v$ and $y$ ensure that one can choose differential representatives on the right-hand side of $(2.5)$ such that the shuffle products are cycles-again, this comes from the calculation. Indeed, as pointed out by the referee, if $N$ is central in $Y$, then the coefficients in (2.4) are trivial and $d_{a, s}^{2}\{[(y \otimes x) \cap v]\}$ may be defined using a homology shuffle product

$$
\cap: H_{a-2}\left(X_{1} ; H_{s+1}(N ; C)\right) \otimes H_{b}\left(X_{2}\right) \rightarrow H_{a+b-2}\left(X ; H_{s+1}(N ; C)\right) .
$$


Proof. Use (2.3) to compute $d_{\mathrm{I}}(y \otimes[x \cap v])=-d_{\mathrm{II}} z$ for $z \in\left(C \otimes_{N} B_{s+1} Y\right) \otimes_{X}$ $B_{a+h-1} X$, where

$$
\begin{aligned}
-z= & \sum_{i \in I}(-1)^{s+i}\left[\left[\left(u\left(x_{1}^{i}\right)\right)^{-1}\right] \cap y\right] \otimes\left[\left[x_{2}^{i}|\cdots| x_{a}^{i}\right] \cap v\right] \\
& +\sum_{j \in J}(-1)^{a+s+j}\left[\left[\left(u\left(v_{1}^{j}\right)\right)^{-1}\right] \cap y\right] \otimes\left[x \cap\left[v_{2}^{j}|\cdots| v_{b}^{j}\right]\right] .
\end{aligned}
$$

Then

$$
\begin{aligned}
d_{\mathrm{I}} z= & \sum_{i}(-1)^{i}\left[\left[\left(u\left(x_{1}^{i}\right)\right)^{-1}\right] \cap y\right] \otimes\left[\partial_{X}\left[x_{2}^{i}|\cdots| x_{a}^{i}\right] \cap v\right] \\
& +\sum_{j}(-1)^{j}\left[\left[\left(u\left(v_{1}^{j}\right)\right)^{-1}\right] \cap y\right] \otimes\left[x \cap \partial_{X}\left[v_{2}^{j}|\cdots| v_{b}^{j}\right]\right] \\
& +\sum_{i} \sum_{j}(-1)^{a+i+j}\left[\left(\left(u\left(x_{1}^{i}\right)^{-1}-1\right)\left[\left(u\left(v_{1}^{j}\right)\right)^{-1}\right]\right.\right. \\
& \otimes\left[\left[x_{2}^{i}|\cdots| x_{a}^{i}\right] \cap\left[v_{2}^{j}|\cdots| v_{b}^{j}\right]\right] .
\end{aligned}
$$

Since $(f-1)[g]-(g-1)[f]=\partial_{Y}\left([f \cap g]+\left[f g \mid f^{-1} g^{-1}\right]-\left[g f \mid f^{-1} g^{-1}\right]\right)+$ $[[f, g]] \sim[[f, g]]$, the expression for $d_{\mathrm{I}} z$ is equivalent to the class in (2.5).

2.5.4 If $x$ and $y$ are as in 2.5.3 with $x=\left[x_{1} \cap x_{2} \cap \cdots \cap x_{a}\right]$, where $\left[x_{i}, x_{j}\right]=1$ $(1 \leqslant i, j \leqslant a)$, then repeated application of $(2.5)$ yields

$$
\begin{aligned}
d_{a, s}^{2}\{y \otimes x\}=\left\{\sum_{\sigma}-\left[\left[\left[\left(u\left(x_{\sigma(1)}\right)\right)^{-1},\left(u\left(x_{\sigma(2)}\right)\right)^{-1}\right]\right]\right.\right. & \cap y] \\
& \left.\otimes\left[x_{\sigma(3)}|\cdots| x_{\sigma(a)}\right]\right\},
\end{aligned}
$$

where $\sigma$ runs over the alternating group $A_{a}$.

3. Low dimensional homology groups of $G_{n}^{k}$. This section inductively looks at low dimensional terms in the spectral sequences

$$
H_{*}\left(G_{n}^{k-1} ; H_{*} M_{n}^{0}\left(t^{k-1}\right)\right) \Rightarrow H_{*} G_{n}^{k}, \quad k>2 .
$$

The first subsection is concerned with $E_{2,0}^{\infty}$ and $E_{0, *}^{\infty}$. Next, the $E_{1,1}^{\infty}$ term in (3.1) is computed, yielding enough information on $\mathrm{H}_{2} G_{n}^{k}$ to estimate $\left(E_{2,1}^{\infty}\right)_{\mathrm{SL}_{n} R}$ recursively. This gives us information on the $\mathrm{SL}_{n} R$-coinvariance of the kernel of $\pi_{*}^{k} \mid H_{3} G_{n}^{k}$. The final subsection looks at $\operatorname{ker}\left(:\left(\pi_{*}^{k} H_{3} G_{n}^{k}\right)_{\mathrm{SL}_{n} R} \rightarrow\left(H_{3} G_{n}^{k-1}\right)_{\mathrm{SL}_{n} R}\right)$.

3.1 LEMMA. (i) $\pi_{*}: H_{1} G_{n}^{k} \rightarrow\left(H_{1} G_{n}^{2} \cong M_{n}^{0}\right)$ is an isomorphism, $k>2$.

(ii) If $k>r \geqslant 2, \operatorname{im}\left(\pi_{*}: H_{2} G_{n}^{k} \rightarrow H_{2} G_{n}^{r}\right)=\operatorname{im}\left(\pi_{*}: H_{2} G_{n}^{r+1} \rightarrow H_{2} G_{n}^{r}\right)$.

(iii) $j_{*}\left(\wedge^{*} M_{n}^{0}\left(t^{k}\right)\right)=0$ in $H_{*} G_{n}^{k+1}$; in particular, $j_{*}\left(H_{2} M_{n}^{0}\left(t^{k}\right)\right)=0$. For torsionfree $R$, in the spectral sequence $(3.1)(k+1), E_{0, *}^{3}=0$ whenever $*>0$ and $k \geqslant 2$.

Proof. Consider (3.1) $(k+1), k \geqslant 2$. For any $\{i, j\} \subset\{1, \ldots, n\}$, fix $m \notin\{i, j\}$. For $\alpha \in R$, define $b_{i j}(\alpha)$ to be $\left[e_{i m}(t) \cap e_{m j}\left(\alpha t^{k-1}\right)\right] \in \mathbf{Z} \otimes_{G_{n}^{k}} B_{2} G_{n}^{k}$. Check that this is a cycle. Let $u: G_{n}^{k} \rightarrow G_{n}^{k+1}$ be a set map section to the reduction; it can be assumed that the elementary matrix $e_{a b}\left(\sum \alpha_{m} t^{m}\right) \in G_{n}^{k}$ is taken to the matrix of the 
same form in $G_{n}^{k+1}$. Apply formula (2.6) of 2.5.4 and the commutator relations 2.3 to compute

$$
\begin{aligned}
-d_{2,0}^{2}\left\{b_{i j}(\alpha)\right\} & =\left\{\left[u\left(e_{i m}(-t)\right), u\left(e_{m j}\left(-\alpha t^{k-1}\right)\right)\right]\right\} \\
& =\left\{\left[e_{i m}(-t), e_{m j}\left(-\alpha t^{k-1}\right)\right]\right\} \\
& =\underline{\alpha}_{i j} \quad \text { if } i \neq j, \text { else }-\underline{\alpha}_{m m}+\underline{\alpha}_{i i} .
\end{aligned}
$$

Therefore $E_{0,1}^{3}=0$. Since $k$ was arbitrary this proves (i).

(ii) $\left\{\left\{b_{i j}(\alpha)\right\}: 1 \leqslant i, j \leqslant n ; \alpha \in R\right\}$ is a set of generators for $H_{2} G_{n}^{k} / \pi_{*} H_{2} G_{n}^{k+1}$. But also $\left\{b_{i j}(\alpha)\right\} \in \operatorname{ker}\left(\pi_{*}: H_{2} G_{n}^{k} \rightarrow H_{2} G_{n}^{k-1}\right)$. Thus in $H_{2} G_{n}^{k-1}, \pi_{*} H_{2} G_{n}^{k+1}=$ $\pi_{*} H_{2} G_{n}^{k}$, which implies (ii).

(iii) Consider (3.1) $(k+1), k \geqslant 2$. By the universal coefficient theorem [M, p. 171] there is an inclusion $H_{2} G_{n}^{k} \otimes H_{a} M_{n}^{0}\left(t^{k}\right) \rightarrow E_{2, a}^{2}$. The proof of (i) shows that $d_{2,0}^{2}\left(H_{2} G_{n}^{k}\right)=M_{n}^{0}\left(t^{k}\right)$. So, by (2.6), $d_{2, a}^{2}\left(E_{2, a}^{2}\right)$ contains the homology product $M_{n}^{0}\left(t^{k}\right) \cap \wedge^{a} M_{n}^{0}\left(t^{k}\right)$, which is just $\wedge^{a+1} M_{n}^{0}\left(t^{k}\right) . \wedge^{2} M_{n}^{0}\left(t^{k}\right)=H_{2} M_{n}^{0}\left(t^{k}\right)$ and, if $R$ is torsion-free, $\wedge^{*} M_{n}^{0}\left(t^{k}\right)=H_{*} M_{n}^{0}\left(t^{k}\right)$ [B, p. 123].

3.2 REMARKS. Let $J_{n}=\operatorname{im}\left(\pi_{*}: H_{2} G_{n}^{k} \rightarrow H_{2} G_{n}^{2}\right), k>2$. (This is independent of $k$, by 3.1(ii).) Then (3.1)(3) contains an exact sequence

$$
J_{n} \stackrel{s}{\rightarrow} \wedge^{2} M_{n}^{0} \stackrel{d^{2}}{\rightarrow} M_{n}^{0} .
$$

Let $L_{n}$ be the associated module defined as $\operatorname{ker}\left([]:, M_{n}^{0} \otimes M_{n}^{0} \rightarrow M_{n}^{0}\right)$, where [, ] is the composite of the homology product with $d^{2}$. The identification (2.2.3) of $M_{n}^{0}$ takes the matrix $a$ to $1+a t \in \mathrm{SL}_{n} R_{2}$. Thus if $a, b \in M_{n}^{0}$, using definition (2.5) $[a, b]$ is the commutator of $1+a t$ and $1+b t$ evaluated in $\mathrm{SL}_{n} R_{3}$. Since $(1+a t)^{-1}$ $\equiv 1-a t+a^{2} t^{2}\left(\bmod t^{3}\right)$, this commutator is readily seen to equal $1+a b t^{2}-b a t^{2}$. As an element of $M_{n}^{0} \cong \operatorname{ker}\left(\pi^{3}: \mathrm{SL}_{n} R_{3} \rightarrow \mathrm{SL}_{n} R_{2}\right)$, this is $a b-b a$; thus $[a, b]$ is the usual Lie bracket.

Let $\Omega \nrightarrow \mathrm{St}(R, R) \stackrel{\phi}{\rightarrow} M_{n}^{0}$ be the universal $\mathrm{SL}_{n} R$-central extension of $M_{n}^{0}[\mathbf{K 4}$, 2.15]. In the Appendix (Lemma A.1(iii)) we show that $\left(M_{n}^{0} \otimes M_{n}^{0}\right) / I \cong \operatorname{St}(R, R) \oplus$ $R$, where $I$ is the $\mathrm{SL}_{n} R$-submodule generated by $\underline{1}_{12} \otimes \underline{1}_{23}+\underline{1}_{43} \otimes \underline{1}_{14}$.

We use this to estimate $\operatorname{ker} \pi_{*}^{k+1} \mid H_{2} G_{n}^{k+1}$, which is the $E_{1,1}^{\infty}$ term in $(3.1)(k+1)$.

3.3 Proposition. (i) There is a commutative diagram

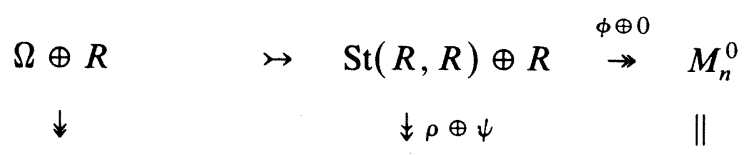

(3.3) $(k+1) \quad \Omega \oplus R /\left(\operatorname{im} d_{3,0}^{2}\right)_{\mathrm{SL}_{n} R} \nrightarrow \operatorname{ker} \pi_{*}^{k+1} \mid H_{2} G_{n}^{k+1} \stackrel{D}{\rightarrow} \quad M_{n}^{0} \quad(k \geqslant 2)$,

where $d_{3,0}^{2}$ is the differential in the spectral sequence $(3.1)(k+1)$ and $D$ is the restriction of the $d_{2,0}^{2}$-differential in $(3.1)(k+2)$.

(ii) $\left(\operatorname{ker} \pi_{*}^{k+1} \mid H_{2} G_{n}^{k+1}\right)_{\mathrm{SL}_{n} R}=\operatorname{im} \psi$.

Proof. Let $u$ be a section to $\pi: G_{n}^{k+1} \rightarrow G_{n}^{k}$ with $u\left(e_{i j}\left(t^{r}\right)\right)=e_{i j}\left(t^{r}\right)$. Use 3.1 to identify $E_{1,1}^{2}$ with $M_{n}^{0} \otimes M_{n}^{0}$. Use the commutator relations 2.3 to check that

$$
x=-\left[e_{12}(t) \cap e_{24}\left(t^{k-1}\right) \cap e_{43}(t)\right]
$$


is a cycle in $\mathbf{Z} \otimes_{G_{n}^{k}} B_{3} G_{n}^{k}$. Use (2.6) to compute

$$
\begin{aligned}
d_{3,0}^{2}\{x\}= & \left\{\left[\left[e_{43}(-t), e_{24}\left(-t^{k-1}\right)\right]\right] \otimes\left[e_{12}(t)\right]\right. \\
& \left.-\left[\left[e_{12}(-t), e_{24}\left(-t^{k-1}\right)\right]\right] \otimes\left[e_{43}(t)\right]\right\} \\
= & \underline{1}_{12} \otimes \underline{1}_{23}+\underline{1}_{43} \otimes \underline{1}_{14} \in H_{1} G_{n}^{k} \otimes M_{n}^{0}\left(t^{k}\right)=M_{n}^{0} \otimes M_{n}^{0} .
\end{aligned}
$$

So $I \subset$ im $d_{3,0}^{2}$.

As in the proof of 3.1(ii), $H_{2} G_{n}^{k+1} / \pi_{*} H_{2} G_{n}^{k+2} \cong M_{n}^{0}$ and is represented in $\operatorname{ker} \pi_{*}^{k+1}=E_{1,1}^{\infty}$. Moreover by the proof of 3.1(i) and Remarks 3.2, in the spectral sequence $(3.1)(k+2), d_{2,0}^{2} \mid \operatorname{ker} \pi_{*}^{k+1}$ is induced by the Lie bracket: $\left(E_{1,1}^{2} \cong M_{n}^{0} \otimes\right.$ $\left.M_{n}^{0}\right) \rightarrow M_{n}^{0}$. According to A.1(ii) if $\operatorname{St}(R, R)$ is identified with $\left(M_{n}^{0} \otimes M_{n}^{0}\right)^{0} / I$, then also $\phi$ is induced by the Lie bracket. This gives the right square of the diagram in the proposition statement. Moreover, because $\operatorname{ker} \pi_{*}^{k+1}=E_{1,1}^{\infty}=\left(M_{n}^{0} \otimes M_{n}^{0}\right) / \operatorname{im} d_{3,0}^{2}$, $\rho \oplus \psi$ has kernel isomorphic to im $d_{3,0}^{2} / I$; as a submodule of $(\Omega \oplus R=\operatorname{ker}(\phi \oplus 0))$, im $d_{3,0}^{2} / I$ has trivial $\mathrm{SL}_{n} R$-action. By Lemma A.1(i) $(I)_{\mathrm{SL}_{n} R}=0$ so $\operatorname{im} d_{3,0}^{2} / I \cong$ (im $\left.d_{3,0}^{2}\right)_{\mathrm{SL}_{n} R}$. This implies the left square of the diagram.

Finally, $(\operatorname{St}(R, R))_{\mathrm{SL}_{n} R}=0[\mathbf{K 4}, 1.7$ and 1.4]; hence part (ii) is implied by the isomorphism $\operatorname{ker} \pi_{*}^{k+1} \cong(\operatorname{St}(R, R) \oplus R) /\left(\operatorname{im} d_{3,0}^{2}\right)_{\mathrm{SL}_{n} R}$.

We next want to estimate $\left(H_{3} G_{n}^{k}\right)_{\mathrm{SL}_{n} R}$. The following lemma will be used in 3.5 to investigate $\left(E_{2,1}^{\infty}\right)_{\mathrm{SL}_{n} R}$.

3.4 Lemma. For $k>2, \mathrm{SL}_{n} R$ acts trivially on $\operatorname{ker}\left(\pi_{*}^{3} \circ \cdots \circ \pi_{*}^{k-1}: \pi_{*}^{k} H_{2} G_{n}^{k} \rightarrow J_{n}\right)$.

Proof. Denote the kernel of $\pi_{*}^{3} \circ \cdots \circ \pi_{*}^{k}$ by $U^{k}$ when it has domain $H_{2} G_{n}^{k}$ and by $\tilde{U}^{k}$ when it has domain $\pi_{*}^{k+1} H_{2} G_{n}^{k+1}$. Use $(3.3)(k)$ to fit the sequence $\operatorname{ker} \pi_{*}^{k} \mid H_{2} G_{n}^{k} \nrightarrow U^{k} \rightarrow \tilde{U}^{k-1}$ into the following diagram, the top row of which is therefore exact. (In (3.5), $D$ is the restriction of the $d_{2,0}^{2}$ differential in the spectral sequence $(3.1)(k+1)$.)

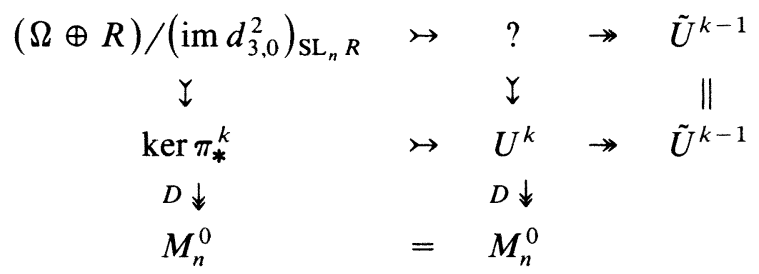

Inductively assume that both the base and fibre modules in the top row of (3.5) have trivial action $\left(\tilde{U}^{2}=0\right)$. Therefore, because $H_{1} \mathrm{SL}_{n} R=0$, ? is also a trivial $\mathrm{SL}_{n} R-$ module. Moreover, the middle column of (3.5) fits into the following diagram, i.e., $?=\tilde{U}^{k}$. This gives the inductive step.

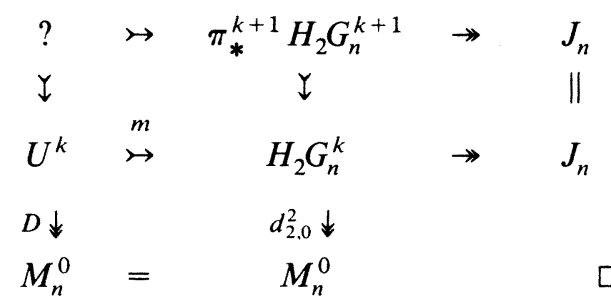


3.5 Proposition. In the spectral sequence $(3.1)(k+1)$ for $k \geqslant 3$,

(i) there is an epimorphism: $R \rightarrow\left(E_{1,2}^{\infty}\right)_{\mathrm{SL}_{n} R}$;

(ii) there is an exact sequence

$$
R \rightarrow\left(\operatorname{ker} d_{2,1}^{2}\right)_{\mathrm{SL}_{n} R} \rightarrow\left(\operatorname{Tor}\left(M_{n}^{0}, M_{n}^{0}\right)\right)_{\mathrm{SL}_{n} R}
$$

hence for some quotient $T$ of $\left(\operatorname{Tor}\left(M_{n}^{0}, M_{n}^{0}\right)\right)_{\mathrm{SL}_{n} R}$, there is an exact sequence

$$
R \rightarrow\left(\left(\operatorname{ker} d_{2,1}^{2} / \operatorname{im} d_{4,0}^{2}\right)=E_{2,1}^{\infty}\right)_{\mathrm{SL}_{n} R} \rightarrow T .
$$

Proof. (i) $E_{1,2}^{\infty}$ is a quotient of $E_{1,2}^{2}=H_{1} G_{n}^{k} \otimes H_{2} M_{n}^{0}\left(t^{k}\right)$. By $3.1, H_{1} G_{n}^{k} \cong M_{n}^{0}$, so $E_{1.2}^{2} \cong M_{n}^{0} \otimes \wedge^{2} M_{n}^{0}$. Use Lemma A.2(iv) to see that $\left(E_{1,2}^{2}\right)_{\mathrm{SL}_{n} R} \cong R$, where $\alpha$ corresponds to the class of $\underline{\alpha}_{12} \otimes \underline{1}_{23} \underline{1}_{31} ;\left(E_{1,2}^{\infty}\right)_{\mathrm{SL}_{n} R}$ is a quotient of this.

(ii) Using the notation and proof of Lemma 3.4, filter $U^{k} \otimes M_{n}^{0}$ as

$$
\tilde{U}^{k} \otimes M_{n}^{0} \rightarrow U^{k} \otimes M_{n}^{0} \stackrel{D \otimes 1}{\rightarrow} M_{n}^{0} \otimes M_{n}^{0},
$$

where in the base group $\underline{\alpha}_{r s} \otimes \underline{\beta}_{u v}$ is the image of $\left\{b_{r s}(\alpha)\right\} \otimes \underline{\beta}_{u v}$ for $\left\{b_{r s}\right\}$ as in 3.1(i)(proof). Hence if $\cap$ is the product $M_{n}^{0} \otimes M_{n}^{0} \rightarrow M_{n}^{0}$, the composite $\cap \circ(D \otimes 1)$ induces an epimorphism $g: M_{n}^{0} \otimes M_{n}^{0} \rightarrow \wedge^{2} M_{n}^{0}=E_{0,2}^{2}$, equivalent to the product map. This has kernel $\Gamma\left(M_{n}^{0}\right)$, the Whitehead gamma group which projects onto $M_{n}^{0} \otimes \mathbf{Z} / 2$ with kernel $S^{2} M_{n}^{0}$, the 2-fold symmetric product of $M_{n}^{0}$ (see, for example, [A]). So $\left(\Gamma\left(M_{n}^{0}\right)\right)_{\mathrm{SL}_{n} R}$ is a quotient of $\left(S^{2} M_{n}^{0}\right)_{\mathrm{SL}_{n} R}=R$ (by Lemma A.2(ii)). Moreover, (3.8) restricts to

$$
\tilde{U}^{k} \otimes M_{n}^{0} \rightarrow \operatorname{ker} \cap \circ(D \otimes 1) \rightarrow \Gamma\left(M_{n}^{0}\right)=\operatorname{ker} g .
$$

Since $\tilde{U}^{k}$ has trivial $\mathrm{SL}_{n} R$-action, $\left(\tilde{U}_{k} \otimes M_{n}^{0}\right)_{\mathrm{SL}_{n} R}=0$; so ker $\cap \circ(D \otimes 1)$ also has $\mathrm{SL}_{n} R$-coinvariance a quotient of $R$. $U_{k}$ is, by definition, $\operatorname{ker}\left(\pi_{*}: H_{2} G_{n}^{k} \rightarrow J_{n}\right)$, and $D$ is the restriction of $d_{2,0}^{2}$. The above shows that the restriction of $d_{2,1}^{2}$ to the image of $U_{k} \otimes M_{n}^{0}$ in $H_{2} G_{n}^{k} \otimes M_{n}^{0}$ is onto $E_{0,2}^{2}$. Hence we have an exact sequence

$$
\operatorname{ker} \cap \circ(D \otimes 1) \rightarrow \operatorname{ker}\left(d_{2,1}^{2} \mid H_{2} G_{n}^{k} \otimes M_{n}^{0}\right) \rightarrow J_{n} \otimes M_{n}^{0} .
$$

Apply $H_{0}\left(\mathrm{SL}_{n} R ;-\right)$ to this and insert result $\left(J_{n} \otimes M_{n}^{0}\right)_{\mathrm{SL}_{n} R}=0$ from Lemma A.2(vi) to see that there is an epimorphism: $R \rightarrow\left(\operatorname{ker} d_{2,1}^{2} \mid H_{2} G_{n}^{k} \otimes M_{n}^{0}\right)_{\mathrm{SL}_{n} R}$.

Finally, the universal coefficient theorem provides a sequence $H_{2} G_{n}^{k} \otimes M_{n}^{0} \hookrightarrow E_{2,1}^{2}$ $\rightarrow \operatorname{Tor}\left(H_{1} G_{n}^{k}, M_{n}^{0}\right)$; because the restriction of $d_{2,1}^{2}$ to $H_{2} G_{n}^{k} \otimes M_{n}^{0}$ is onto $E_{0,2}^{2}$, there is a sequence

$$
\operatorname{ker} d_{2,1}^{2} \mid H_{2} G_{n}^{k} \otimes M_{n}^{0} \rightarrow \operatorname{ker} d_{2,1}^{2} \rightarrow \operatorname{Tor}\left(H_{1} G_{n}^{k}, M_{n}^{0}\right) .
$$

Apply $H_{0}\left(\mathrm{SL}_{n} R ;-\right)$ to this and identify $H_{1} G_{n}^{k}$ with $M_{n}^{0}$ to get (3.6).

3.6 Proposition. Whenever $k>2$, there is an exact sequence

$$
H_{1}\left(\mathrm{SL}_{n} R ; I\right) \stackrel{\partial_{k}}{\rightarrow}\left(\pi_{*}^{k} H_{3} G_{n}^{k}\right)_{\mathrm{SL}_{n} R} \stackrel{\iota_{*}}{\rightarrow}\left(H_{3} G_{n}^{k-1}\right)_{\mathrm{SL}_{n} R} \rightarrow\left(\mathrm{im} d_{3,0}^{2}\right)_{\mathrm{SL}_{n} R}
$$

where $I$ is the $\mathrm{SL}_{n} R$-submodule of $M_{n}^{0} \otimes M_{n}^{0}$ generated by $\underline{1}_{12} \otimes \underline{1}_{23}+\underline{1}_{43} \otimes \underline{1}_{14}$.

Proof. Consider the spectral sequence $(3.1)(k)$. There can be no transgressions from $E_{3,0}^{3}$, as $E_{0,2}^{3}=0$ (proof of Proposition 3.1(iii)). Thus there is a sequence $\pi_{*}^{k} H_{3} G_{n}^{k} \nrightarrow H_{3} G_{n}^{k-1} \rightarrow \operatorname{im} d_{3,0}^{2}$. Apply $H_{*}\left(\mathrm{SL}_{n} R ;-\right)$ to this to get (3.9) except that $\iota_{*}$ has kernel $\partial\left(H_{1}\left(\mathrm{SL}_{n} R ;\right.\right.$ im $\left.\left.d_{3,0}^{2}\right)\right)$. 
Identify $H_{1} G_{n}^{k-1} \otimes M_{n}^{0}\left(t^{k-1}\right)$ with $M_{n}^{0} \otimes M_{n}^{0}$ via 3.1(i) and identify $I$ with $\left\{d_{3,0}^{2}(x)\right\}$ as in 3.3(proof). As in the last part of the proof of 3.3, im $d_{3,0}^{2} / I$ is the trivial $\mathrm{SL}_{n} R$-module (im $\left.d_{3,0}^{2}\right)_{\mathrm{SL}_{n} R}$. So $H_{1}\left(\mathrm{SL}_{n} R\right.$; im $\left.d_{3,0}^{2} / I\right)=0$, implying an epimorphism: $H_{1}\left(\mathrm{SL}_{n} R ; I\right) \rightarrow H_{1}\left(\mathrm{SL}_{n} R ;\right.$ im $\left.d_{3,0}^{2}\right)$. $\partial_{k}$ is the composite of this epimorphism and $\partial$.

4. $K_{3}$ of truncated polynomial rings over the integers. The first three subsections refer to general rings $R$ and concern the $E_{1,2}^{*}$ terms in the spectral sequences

$$
{ }^{k} E_{* *}^{2}=H_{*}\left(\mathrm{SL}_{n} R ; H_{*} G_{n}^{k}\right) \Rightarrow H_{*} \mathrm{SL}_{n} R_{k}, \quad k \geqslant 2 \text {. }
$$

Lemmas 4.4 and 4.5 determine $H_{1}\left(\mathrm{SL}_{n} \mathrm{Z} ; \wedge^{2} M_{n}^{0}\right)$ and $H_{1}\left(\mathrm{SL}_{n} \mathrm{Z} ; I\right)$. This and earlier work yield the main theorem which computes $H_{3} \mathrm{SL}_{n} \mathbf{Z}[t] /\left(t^{k}\right), k \geqslant 2$.

4.1 Proposition. For $k>2$ there is an exact sequence

$$
\left(R+N_{k}\right) / R \rightarrow H_{1}\left(\mathrm{SL}_{n} R ; H_{2} G_{n}^{k}\right) \stackrel{\pi_{*}}{\rightarrow} H_{1}\left(\mathrm{SL}_{n} R ; J_{n} \equiv \pi_{*}^{3} H_{2} G_{n}^{3}\right),
$$

where $N_{k}$ is the $\mathrm{SL}_{n} R$-coinvariance of the image of the $d_{3,0}^{2}$-differential in the spectral sequence (3.1)( $k)$, and $R+N_{k}=R$ if $H_{1}\left(\mathrm{SL}_{n} R ; M_{n}^{0}\right)=0$ (see Proposition 3.3).

Proof. Proposition 3.3 provides a sequence $N_{k} \rightarrow \Omega \oplus R \rightarrow \operatorname{ker} \pi_{*}^{k} \rightarrow M_{n}^{0}$, in which $\left(\operatorname{ker} \pi_{*}^{k}\right)_{\mathrm{SL}_{n} R} \cong \operatorname{im} R=\left(R+N_{k}\right) / N_{k}$ (implying the splitting $(\Omega \oplus R) / N_{k} \cong$ $\left.\left(\left(R+N_{k}\right) / N_{k}\right) \oplus(\Omega \oplus R) /\left(R+N_{k}\right)\right)$. Application of $H_{*}\left(\mathrm{SL}_{n} R ;-\right)$ to the sequence $(3.3)(k)$ yields

$$
\begin{aligned}
H_{1}\left(\mathrm{SL}_{n} R ; \operatorname{ker} \pi_{*}^{k}\right) & \mapsto H_{1}\left(\mathrm{SL}_{n} R ; M_{n}^{0}\right) \\
& =\Omega \rightarrow(\Omega \oplus R) / N_{k} \rightarrow\left(\operatorname{ker} \pi_{*}^{k}\right)_{\mathrm{SL}_{n} R}=\left(R+N_{k}\right) / N_{k}
\end{aligned}
$$

Thus $H_{1}\left(\mathrm{SL}_{n} R ; \operatorname{ker} \pi_{*}^{k}\right) \cong\left(R+N_{k}\right) / R$; its image under the map induced by inclusion is the kernel of

$$
\pi_{*}:\left(H_{1}\left(\mathrm{SL}_{n} R ; H_{2} G_{n}^{k}\right) \rightarrow H_{1}\left(\mathrm{SL}_{n} R ; \pi_{*}^{k} H_{2} G_{n}^{k}\right) \stackrel{\sigma}{\rightarrow} H_{1}\left(\mathrm{SL}_{n} R ; J_{n}\right)\right)
$$

where the injection $\sigma$ is implied by Lemma 3.4.

4.2 Proposition. If $H_{2}\left(\mathrm{SL}_{n} R ; M_{n}^{0}\right)=0$ and $s: J_{n} \nrightarrow \wedge^{2} M_{n}^{0}$ is as in (3.2), then there is an injection

$$
s_{*}: H_{1}\left(\mathrm{SL}_{n} R ; J_{n}\right) \rightarrow H_{1}\left(\mathrm{SL}_{n} R ; H_{2} G_{n}^{2}=\wedge^{2} M_{n}^{0}\right) .
$$

If $H_{1}\left(\mathrm{SL}_{n} R ; M_{n}^{0}\right)=0, s_{*}$ is an isomorphism.

Proof. Apply $H_{*}\left(\mathrm{SL}_{n} R ;-\right)$ to the exact sequence $J_{n} \stackrel{s}{\rightarrow} \wedge^{2} M_{n}^{0} \rightarrow M_{n}^{0}$ of (3.2) and use the assumption.

4.3 Proposition. Suppose $H_{i}\left(\mathrm{SL}_{n} R ; M_{n}^{0}\right)=0$ for $i=1$, 2. Denote $E_{* *}^{*}$ terms in the spectral sequence $(4.1)(k)$ by ${ }^{k} E_{* *}^{*}, k \geqslant 2$. Then $\pi_{*}:{ }^{k} E_{1,2}^{\infty} \rightarrow{ }^{2} E_{1,2}^{\infty}$ is an injection.

Proof. By 4.1 and the assumption, ${ }^{k} E_{1,2}^{2}$ injects into $H_{1}\left(\mathrm{SL}_{n} R ; J_{n}\right)$ which by 4.2 is isomorphic to ${ }^{2} E_{1,2}^{2}$. If $j<2, \pi_{*}: H_{j} G_{n}^{k} \rightarrow H_{j} G_{n}^{2}$ is an isomorphism (Lemma 3.1) so that $\pi_{*}:{ }^{k} E_{1,2}^{r} \rightarrow{ }^{2} E_{1,2}^{r}$ is an injection for $r \geqslant 2$. 
The remainder of this section is devoted to computing $K_{3} \mathrm{Z}[t] /\left(t^{k}\right)$; two preliminary lemmas are required. Let $M_{n}^{0} R$ denote the submodule of zero-trace matrices in the $\mathrm{SL}_{n} R$-module $M_{n} R$ of $n \times n$ matrices over $R$.

4.4 LEMMA. If $n$ is large and $p$ is any prime,

(i) $H_{1}\left(\mathrm{SL}_{n} \mathbf{Z} / p^{2} ; M_{n}^{0} \mathbf{Z} / p \otimes M_{n}^{0} \mathbf{Z} / p\right)=0 ; H_{1}\left(\mathrm{SL}_{n} \mathbf{Z} ; M_{n}^{0} \mathbf{Z} / p \otimes M_{n}^{0} \mathbf{Z} / p\right)=0 ;$ $H_{1}\left(\mathrm{SL}_{n} \mathbf{Z} ; M_{n}^{0} \mathbf{Z} \otimes M_{n}^{0} \mathbf{Z}\right)=0$

(ii) $H_{1}\left(\mathrm{SL}_{n} \mathbf{Z} ; I\right)=0$, where $I$ is as in 3.2 .

Proof. (i) Take $n$ large and prime to $p$ and to $p-1$. Consider the spectral sequence

$$
E_{* *}^{2}(C)=H_{*}\left(\mathrm{SL}_{n} \mathbf{Z} / p ; H_{*}\left(M_{n}^{0} \mathbf{Z} / p ; C\right)\right) \Rightarrow H_{*}\left(\mathrm{SL}_{n} \mathbf{Z} / p^{2} ; C\right)
$$

firstly with $C=M_{n}^{0} \mathbf{Z} / p$. The proof of [ALSS, part 1, VI, 1.1] demonstrates an isomorphism which is dual to $d_{2,0}^{2}$. Moreover, $M_{n}^{0} \mathbf{Z} / p$ may be viewed as a direct summand of $M_{n}^{0} \mathbf{Z} / p \otimes M_{n}^{0} \mathbf{Z} / p$ using either of the inclusions

$$
\Phi: \underline{\boldsymbol{\alpha}}_{i j} \mapsto\left[\sum_{k=1}^{n} \underline{\boldsymbol{\alpha}}_{i k} \otimes \underline{1}_{k j}\right] \text { or } \quad \Phi^{T}: \underline{\boldsymbol{\alpha}}_{i j} \mapsto\left[\sum_{k=1}^{n}-\underline{1}_{k j} \otimes \underline{\boldsymbol{\alpha}}_{i k}\right],
$$

where if $a \otimes b \in M_{n} \mathbf{Z} / p \otimes M_{n} \mathbf{Z} / p,[a \otimes b]$ is its image in $M_{n}^{0} \mathbf{Z} / p \otimes M_{n}^{0} \mathbf{Z} / p$ under the canonical projection $\left(M_{n} \mathbf{Z} / p\right)^{\otimes 2}=\left(M_{n}^{0} \mathbf{Z} / p \oplus \mathbf{Z} / p\right)^{\otimes 2} \rightarrow\left(M_{n}^{0} \mathbf{Z} / p\right)^{\otimes 2}$. (Since $p+n, \Phi$ and $\Phi^{T}$ are split by the Lie bracket.)

Now

$$
\begin{aligned}
E_{0,1}^{2}\left(M_{n}^{0} \mathbf{Z} / p \otimes M_{n}^{0} \mathbf{Z} / p\right) & \cong\left[\left(M_{n}^{0} \mathbf{Z} / p\right)^{\otimes 3}\right]_{\mathrm{SL}_{n} \mathbf{Z} / p} \\
& =\Phi_{*}\left(E_{0,1}^{2}\left(M_{n}^{0} \mathbf{Z} / p\right)\right) \oplus \Phi_{*}^{T}\left(E_{0,1}^{2}\left(M_{n}^{0} \mathbf{Z} / p\right)\right),
\end{aligned}
$$

from A.2(i) and (iii). Therefore by naturality,

$$
E_{0,1}^{2}\left(M_{n}^{0} \mathbf{Z} / p \otimes M_{n}^{0} \mathbf{Z} / p\right)=d_{2,0}^{2}\left(\Phi_{*}\left(E_{2,0}^{2}\left(M_{n}^{0} \mathbf{Z} / p\right)\right)+\Phi_{*}^{T}\left(E_{2,0}^{2}\left(M_{n}^{0} \mathbf{Z} / p\right)\right)\right) .
$$

The proof of the first equality in part (i) is completed by recalling that

$$
\left(E_{1,0}^{2}\left(M_{n}^{0} \mathbf{Z} / p \otimes M_{n}^{0} \mathbf{Z} / p\right) \equiv H_{1}\left(\mathrm{SL}_{n} \mathbf{Z} / p ; M_{n}^{0} \mathbf{Z} / p \otimes M_{n}^{0} \mathbf{Z} / p\right)\right)=0,
$$

dually to [ALSS, part 1, II, 1.4]. Given this, Kassel [K2, 3.4] asserts that the second equality holds. This then implies that multiplication by $p$ is an epimorphism on $H_{1}\left(\mathrm{SL}_{n} \mathbf{Z} ; M_{n}^{0} \mathbf{Z} \otimes M_{n}^{0} \mathbf{Z}\right)$ which, since we are dealing with a finitely generated abelian group (e.g. [B, p. 217]) means the group is torsion with trivial $p$-component. However, for large enough $n$ the inclusion: $M_{n}^{0} \mathbf{Z} \rightarrow M_{n+m}^{0} \mathbf{Z}$ induces isomorphisms

$$
H_{i}\left(\mathrm{SL}_{n} \mathbf{Z} ; M_{n}^{0} \mathbf{Z} \otimes M_{n}^{0} \mathbf{Z}\right) \rightarrow H_{i}\left(\mathrm{SL}_{n+m} \mathbf{Z} ; M_{n+m}^{0} \mathbf{Z} \otimes M_{n+m}^{0} \mathbf{Z}\right), \quad m \geqslant 1
$$

(e.g. [VK, §5]). Thus for $n$ sufficiently large, $H_{1}\left(\mathrm{SL}_{n} \mathbf{Z} ; M_{n}^{0} \mathbf{Z} \otimes M_{n}^{0} \mathbf{Z}\right)$ has no $p$-component for any prime $p$, giving (i).

(ii) If $I \subset M_{n}^{0} \mathbf{Z} \otimes M_{n}^{0} \mathbf{Z}$ is as in $3.2, I \otimes \mathbf{Z} / p \subset M_{n}^{0} \mathbf{Z} / p \otimes M_{n}^{0} \mathbf{Z} / p$ is the $\mathrm{SL}_{n} \mathbf{Z} / p$-module generated by $\underline{1}_{12} \otimes \underline{1}_{23}+\underline{1}_{43} \otimes \underline{1}_{14}$, and equation (A.7) (see A.2(iii) proof) shows that $\left(I \otimes M_{n}^{0} \mathbf{Z} / p\right)_{\mathbf{S L}_{n} \mathbf{Z} / p}=\mathbf{Z} / p$, generated by the class of $\underline{1}_{12} \otimes \underline{1}_{23}$ $\otimes \underline{1}_{31}+\underline{1}_{43} \otimes \underline{1}_{14} \otimes \underline{1}_{31}$. Embed $M_{n}^{0} \mathbf{Z} / p$ into $I \otimes \mathbf{Z} / p$ with the map $\Phi-\Phi^{T}$, then argue as for part (i). 
4.5 LEMMA, If $n$ is large and $p$ is any odd prime, $H_{1}\left(\mathrm{SL}_{n} \mathbf{Z} ; \wedge^{2} M_{n}^{0} \mathbf{Z} / p\right)=0$; $H_{1}\left(\mathrm{SL}_{n} \mathbf{Z} ; \wedge^{2} M_{n}^{0} \mathbf{Z} / 2\right)=\mathbf{Z} / 2 ; H_{1}\left(\mathrm{SL}_{n} \mathbf{Z} ; \wedge^{2} M_{n}^{0} \mathbf{Z}\right)=0$.

Proof. If $p$ is odd, $\wedge^{2} M_{n}^{0} \mathbf{Z} / p$ is a direct summand of $M_{n}^{0} \mathbf{Z} / p \otimes M_{n}^{0} \mathbf{Z} / p$, so the first equality follows from $4.4(\mathrm{i})$.

Define $\Phi^{\prime}: M_{n}^{0} \mathbf{Z} / 2 \rightarrow \wedge^{2} M_{n}^{0} \mathbf{Z} / 2$ to be the composite of $\Phi$ (defined in (4.5)) with the homology product on $H_{*} M_{n}^{0} \mathbf{Z} / 2$. The map $\Phi_{*}^{\prime}: E_{0,1}^{2}\left(M_{n}^{0} \mathbf{Z} / 2\right) \rightarrow$ $E_{0.1}^{2}\left(\wedge^{2} M_{n}^{0} \mathbf{Z} / 2\right)$ between terms in the spectral sequences (4.4) is an isomorphism, by A.2. Arguing as in the proof of 4.4 , we conclude that $E_{0,1}^{3}\left(\wedge^{2} M_{n}^{0} \mathbf{Z} / 2\right)=0$. Thus the reduction epimorphism: $H_{1}\left(\mathrm{SL}_{n} \mathbf{Z} / 4 ; \wedge^{2} M_{n}^{0} \mathbf{Z} / 2\right) \rightarrow H_{1}\left(\mathrm{SL}_{n} \mathbf{Z} / 2 ; \wedge^{2} M_{n}^{0} \mathbf{Z} / 2\right)$ is injective; its image is identified as $\mathbf{Z} / 2$ in [ALSS, part 3, 9.16]. Again apply the Kassel result $[\mathbf{K 2}, 3.4]$ to conclude that $H_{1}\left(\mathrm{SL}_{n} \mathbf{Z} ; \wedge^{2} M_{n}^{0} \mathbf{Z} / 2\right)=\mathbf{Z} / 2$.

Now $\mathrm{SL}_{n} \mathbf{Z}$ acts on $\wedge^{2} M_{n}^{0} \mathbf{Z} / 2$ via reduction to $\mathrm{SL}_{n} \mathbf{Z} / 2$. Thus we can use Lemma A.2 to see that each of the terms in the exact sequence $\wedge^{2} M_{n}^{0} \mathbf{Z} \stackrel{2}{\rightarrow} \wedge^{2} M_{n}^{0} \mathbf{Z} \rightarrow$ $\wedge^{2} M_{n}^{0} \mathbf{Z} / 2$ has $\mathrm{SL}_{n} \mathbf{Z}$-coinvariance $\mathbf{Z} / 2$. In the long exact sequence obtained on application of $H_{*}\left(\mathrm{SL}_{n} \mathbf{Z} ;-\right)$ to this coefficient sequence, the connecting homomorphism $H_{1}\left(\mathrm{SL}_{n} \mathbf{Z} ; \wedge^{2} M_{n}^{0} \mathbf{Z} / 2\right) \rightarrow \mathbf{Z} / 2$ must therefore be onto, hence injective.

So multiplying the coefficients in $H_{1}\left(\mathrm{SL}_{n} \mathbf{Z} ; \wedge^{2} M_{n}^{0} \mathrm{Z}\right)$ by two results in an onto map. Since we are dealing with a finitely generated group, it must be an odd torsion group. The first equality in the statement of the lemma then implies it is trivial.

4.6 THEOREM. If $k \geqslant 2$ and $n$ is large there are exact sequences

$$
\mathbf{Z} \oplus \mathbf{Z} \mapsto H_{3} \mathrm{SL}_{n} \mathbf{Z}_{k+1} \stackrel{\pi_{*}^{k+1}}{\rightarrow} H_{3} \mathrm{SL}_{n} \mathbf{Z}_{k}=\mathbf{Z}^{k-1} \oplus \mathbf{Z} / 24 \rightarrow \mathbf{Z},
$$

and

$$
\mathbf{Z} \oplus \mathbf{Z} \oplus \mathbf{Z} / 2 \stackrel{1 \oplus 1 \oplus \iota}{\rightarrow} K_{3} \mathbf{Z}_{k+1}=K_{3} \mathbf{Z} \oplus \mathbf{Z}^{k} \oplus U_{k+1} \rightarrow K_{3} \mathbf{Z}_{k} \rightarrow \mathbf{Z}
$$

where $U_{k}$ is an elementary 2-group of rank at most $[k / 2]$ and $\iota=0$ if $k$ is even. (Here $\mathbf{Z}_{k}$ denotes $\mathbf{Z}[t] /\left(t^{k}\right)$.)

Proof. Take $R=\mathbf{Z}$. If $n>10, H_{2}\left(\mathrm{SL}_{n} \mathbf{Z} ; M_{n}^{0}\right)=0[\mathbf{K} 1]$, and of course, $\Omega \equiv$ $\Omega_{\mathbf{Z} / \mathbf{Z}}^{1}=0 . H_{1}\left(\mathrm{SL}_{n} \mathbf{Z} ; \wedge^{2} M_{n}^{0}\right)=0$ by Lemma 4.5 , so that by Propositions 3.1 and 4.3 , in the spectral sequence $(4.1)(k), k \geqslant 2,{ }^{k} E_{1,2}^{\infty}=0$ and ${ }^{k} E_{2,1}^{2}=0$. Thus there is an epimorphism, induced by the inclusion of $G_{n}^{k}$ into $\mathrm{SL}_{n} R_{k}$,

$$
i_{*}^{k}:\left(H_{3} G_{n}^{k}\right)_{\mathrm{SL}_{n} R} \rightarrow H_{3} \mathrm{SL}_{n} R_{k} / H_{3} \mathrm{SL}_{n} R
$$

Take $k \geqslant 3$. By (4.6), $\operatorname{ker} \pi_{*}^{k} \mid H_{3} \mathrm{SL}_{n} R_{k}$ is the quotient of

$$
V_{k}=\operatorname{ker}\left(i_{*}^{k-1} \circ \pi_{*}^{k}:\left(H_{3} G_{n}^{k}\right)_{\mathrm{SL}_{n} R} \rightarrow H_{3} \mathrm{SL}_{n} R_{k-1}\right)
$$

by $\operatorname{ker} i_{*}^{k}$. Use (3.9) and Lemma 4.4 to identify

$$
\operatorname{ker}\left(:\left(\pi_{*}^{k} H_{3} G_{n}^{k}\right)_{\mathrm{SL}_{n} R} \rightarrow\left(H_{3} G_{n}^{k-1}\right)_{\mathrm{SL}_{n} R}\right)=0
$$

then use this fact in constructing the exact sequence

$$
\left(\operatorname{ker} \pi_{*}^{k} \mid H_{3} G_{n}^{k}\right)_{\mathrm{SL}_{n} R} \stackrel{\sigma}{\rightarrow} V_{k} \rightarrow \operatorname{ker}\left(i_{*}^{k-1} \mid\left(H_{3} G_{n}^{k-1}\right)_{\mathrm{SL}_{n} R}\right) .
$$


By Propositions 3.5 and 3.1, im $\sigma$ has at most two generators. For the case $k=2$, we have $H_{3} \mathrm{SL}_{n} \mathbf{Z}=\mathbf{Z} / 24$ (e.g. [ALSS, part 1, VI]) and by A.2(v), $\left(H_{3} G_{n}^{2}\right)_{\mathrm{SL}_{n} R}=\mathbf{Z}$. So $H_{3} \mathrm{SL}_{n} R_{2} \leqslant \mathbf{Z} \oplus \mathbf{Z} / 24$.

Now suppose $n=\infty$. Theorem 2.1 of [A] proposes an exact sequence

$$
K_{2} R_{r} \otimes \mathbf{Z} / 2 \rightarrow K_{3} R_{r} \rightarrow H_{3} \mathrm{SL} R_{r} \quad(r \geqslant 1) .
$$

There is a canonical epimorphism: $K_{3} \mathbf{Z}_{k} \rightarrow \mathbf{Z}^{k-1}$, with $K_{3} \mathbf{Z}_{k-1} / \pi_{*} K_{3} \mathbf{Z}_{k}=\mathbf{Z}$; hence $H_{3} \mathrm{SL}_{n} R_{2}=\mathbf{Z} \oplus \mathbf{Z} / 24$ and if $k \geqslant 3$ there is an induced epimorphism: $\operatorname{ker}\left(K_{3} \mathbf{Z}_{k} \rightarrow K_{3} \mathbf{Z}_{k-1}\right) \rightarrow \mathbf{Z} \oplus \mathbf{Z}$ (Stienstra [S, Theorem 1.13]). As $K_{3} R_{k}=H_{3}$ SL $R_{k}$ off torsion, an inductive argument from the initial case $k=3$ shows that in (4.7), $\operatorname{ker} i_{*}^{k-1}=0$ and $\operatorname{im} \sigma=\mathbf{Z} \oplus \mathbf{Z}=\operatorname{ker} \pi_{*}^{k} \mid H_{3} \mathrm{SL}_{n} R_{k}$. The first of the exact sequences in the theorem statement follows.

The theorem for general $n$ is a consequence of the stability of the generators. The $K$-theory result is implied by the $n=\infty$ part of the earlier statement, and (4.8), together with the identifications [Ro, Theorem 4] $K_{2} \mathbf{Z}_{k}=\mathbf{Z} / 2 \oplus\left(\oplus_{i=2}^{k} \mathbf{Z} / i\right)$ and $K_{3} \mathbf{Z}=\mathbf{Z} / 48$.

5. $K_{3}$ of truncated polynomial rings over finite fields. Throughout this section, $R$ is a finite field of characteristic $p$ greater than 2, and $n$ is a large integer. We obtain $K_{3} R_{k}$ by using a van der Kallen-Stienstra result in conjunction with an estimate of the quotient of group orders $\# K_{3} R_{k+1} / \# K_{3} R_{k}$. The latter is obtained from the spectral sequences and associated maps:

$(5.2)(k+1)$

$$
\begin{gathered}
H_{*}\left(\mathrm{SL}_{n} R_{k} ; H_{*} M_{n}^{0}\left(t^{k}\right)\right) \quad \Rightarrow \\
\uparrow\left(i_{*}, 1\right)
\end{gathered}
$$$$
H_{*}\left(G_{n}^{k} ; H_{*} M_{n}^{0}\left(t^{k}\right)\right)
$$

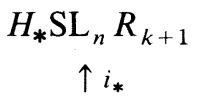

$$
H_{*} G_{n}^{k+1}
$$

$(k \geqslant 2)$.

The first subsection reviews various groups $H_{i}\left(\mathrm{SL}_{n} R ; H_{j} M_{n}^{0}\right)$ for $j \leqslant 3$. The next four subsections look at $E_{* *}^{*}$ terms of total degree 3 in the spectral sequence (5.1) $(k+1)$, from which $\operatorname{ker} \pi_{*}^{k+1} \mid H_{3} \mathrm{SL}_{n} R_{k+1}$ is estimated in 5.6. Proposition 5.7 determines $H_{3} \mathrm{SL}_{n} R_{k} / \pi_{*} H_{3} \mathrm{SL}_{n} R_{k+1}$ then obtains the desired quotient of group orders. Finally, the computation of $K_{2}\left(R_{q},\left(t^{k}\right)\right)$ in [VKS] is invoked to give the main theorem, 5.9.

Our constraints on the dimension $n$ are introduced by Proposition 5.1. The results of Lluis [ALSS], phrased in terms of the general linear group $\mathrm{GL}_{n} R$ and the full matrix group $M_{n} R$, hold for large $n$. By restricting to $n$ relatively prime to $p$ and $p-1$, his results are simply expressed in terms of the respective direct summands $\mathrm{SL}_{n} R$ and $M_{n}^{0}$; however, applying stability theorems such as those in [VK], we need only assume $n$ to be "sufficiently large".

5.1 Proposition. (i) $H_{i}\left(\mathrm{SL}_{n} R ; M_{n}^{0}\right)=0=H_{i}\left(\mathrm{SL}_{n} R ; \bigwedge^{2} M_{n}^{0}\right)$ for $i=0$ or 1 .

(ii) $H_{0}\left(\mathrm{SL}_{n} R ; H_{3} M_{n}^{0}\right)=\left(S^{2} M_{n}^{0}\right)_{\mathrm{SL}_{n} R} \oplus\left(\bigwedge^{3} M_{n}^{0}\right)_{\mathrm{SL}_{n} R}=R \oplus R$.

(iii) $H_{1}\left(\mathrm{SL}_{n} R ; M_{n}^{0} \otimes M_{n}^{0}\right)=0$.

(iv) $H_{2}\left(\mathrm{SL}_{n} R ; M_{n}^{0}\right)=R$.

Proof. (i), (ii) and (iv) are derived from Lluis [ALSS]. The proof of (iii) is analogous to that used in [ALSS] to prove $H_{1}\left(\mathrm{SL}_{n} R ; \wedge^{2} M_{n}^{0}\right)=0$. 
5.2 Lemma. For $k \geqslant 2, R \cong H_{1}\left(\mathrm{SL}_{n} R_{k} ; \wedge^{2} M_{n}^{0}\left(t^{k}\right)\right)$.

Proof. $\mathrm{SL}_{n} R_{k}=\mathrm{SL}_{n} R \ltimes G_{n}^{k}$, so that for any coefficient module $C$,

$$
H_{1}\left(\mathrm{SL}_{n} R_{k} ; C\right)=H_{1}\left(\mathrm{SL}_{n} R ; C\right) \oplus\left(H_{1}\left(G^{k} ; C\right)\right)_{\mathrm{SL}_{n} R}
$$

Set $C=\wedge^{2} M_{n}^{0}$ and use 5.1(i), the isomorphism $\pi_{*}: H_{1} G_{n}^{k} \rightarrow M_{n}^{0}$ of 3.1(i), and A.2(iv).

5.3 Lemma. For $k \geqslant 2, j_{*} H_{3} M_{n}^{0}\left(t^{k}\right)=0$ in $H_{*} G_{n}^{k+1}$ and hence $i_{*}^{\circ} j_{*} H_{3} M_{n}^{0}\left(t^{k}\right)$ $=0$ in $H_{*} \mathrm{SL}_{n} R_{k+1}$.

Proof. The case $k=2$ is [ALS, 2.7 and 2.8(iii)]. Take $k \geqslant 3$. Then according to Proposition 3.1(iii), $E_{0.1}^{3}=0$ in the spectral sequence $(5.2)(k+1)$. Further, it implies $H_{2} G_{n}^{k} / \pi_{*} H_{2} G_{n}^{k+1}$ is represented in $E_{1,1}^{\infty}$.

If $\beta$ is the homology Bockstein associated to the coefficient sequence $\mathbf{Z} \rightarrow \mathbf{Z} \rightarrow$ $\mathbf{Z} / p$, and $s \geqslant 1, \beta H_{s+1}\left(M_{n}^{0} ; \mathbf{Z} / p\right)=H_{s} M_{n}^{0}$ so the lemma is proved by showing that $j_{*} H_{*}\left(M_{n}^{0} ; \mathbf{Z} / p\right)=0$ in $H_{*}\left(G_{n}^{k} ; \mathbf{Z} / p\right)$, or, dually, that $j^{*} H^{*}\left(G_{n}^{k} ; \mathbf{Z} / p\right)=0$. Consider the cohomology spectral sequence

$$
H^{*}\left(G_{n}^{k} ; \mathbf{Z} / p\right) \otimes H^{*}\left(M_{n}^{0} ; \mathbf{Z} / p\right) \Rightarrow H^{*}\left(G_{n}^{k+1} ; \mathbf{Z} / p\right), \quad k>2
$$

Denote terms in this sequence by ${ }^{k+1} E_{*}^{* *}$.

Dually to the homology results, ${ }^{k+1} E_{3}^{0,1}=0$ and $i m d_{2}^{0,1}$ lies in a direct summand of $H^{2}\left(G_{n}^{k} ; \mathbf{Z} / p\right)$ which is represented in ${ }^{k} E_{\infty}^{1,1}$. (In (3.3)(k), the map $D$ induced by the homology differential is split since $H_{1}\left(\mathrm{SL}_{n} R ; M_{n}^{0}\right) \equiv \Omega \equiv 0$.)

Because $d_{2}^{0, *} \mid \wedge^{*} M_{n}^{0 \#}$ is a derivation with image in the $\mathbf{Z} / p$-vector space $H^{2} G_{n}^{k} \otimes$ $\wedge^{*} M_{n}^{0 \#}$, the injectivity of $d_{2}^{0,1}$ implies that of $d_{2}^{0, *} \mid \wedge^{*} M_{n}^{0 \#}$. Now suppose $\beta^{\#}$ is the cohomology Bockstein associated to the coefficient sequence $\mathbf{Z} / p \rightarrow \mathbf{Z} / p^{2} \rightarrow \mathbf{Z} / p$. Since $M_{n}^{0}$ is a $\mathbf{Z} / p$-vector space, $H^{*}\left(M_{n}^{0} ; \mathbf{Z} / p\right)=\Lambda^{*} M_{n}^{0 \#} \otimes S^{*}\left(\beta^{\#} M_{n}^{0 \#}\right)$. Thus $\left(E_{3}^{0, *}=\operatorname{ker} d_{2}^{0, *}\right)=S^{*}\left(\beta^{\#} M_{n}^{0 \#}\right)$. We want to show that $d_{3}^{0,2} \beta^{\#} M_{n}^{0 \#} \cong M_{n}^{0 \#}$.

Observe first that the connecting homomorphism $\beta^{\#}$ commutes with spectral sequence differentials which are transgressive. (This can be seen from the geometric description of the transgression of e.g. [M, p. 335].) So $d_{3}^{0,2} \beta^{\#} M_{n}^{0 \#}=\beta^{\#} d_{2}^{0,1} M_{n}^{0 \#}$ modulo im $d_{2}^{1,1}$.

We claim next that $\beta^{\#} d_{2}^{0,1} M_{n}^{0 \#}$ is represented in ${ }^{k} E_{\infty}^{1,2}$ by a submodule isomorphic to $M_{n}^{0 \#}$. To see this, at the cochain level apply the Cartan formula, $\beta^{\#}(a \otimes b)$ $=\left(\beta^{\#} a\right) \otimes b \pm a \otimes \beta^{\#} b$, which is a consequence of the definition of the connecting homomorphism in terms of the cochain boundary maps, which are derivations (see e.g. [M, pp. 190, 52]). Note that here we are dealing with the cochain bicomplex underlying the spectral sequence $(5.3)(k)$, not $(5.3)(k+1)$. If $\tilde{x} \neq 0$ in ${ }^{k+1} E_{2}^{0,1}$ and $x \neq 0$ represents $d_{2}^{0,1} \tilde{x}$ in ${ }^{k} E_{2}^{1,1}$, then $\left(1 \otimes \beta^{\#}\right) x$ represents $\beta^{\#} d_{2}^{0,1} \tilde{x}$ in ${ }^{k} E_{2}^{1,2}$, and is nonzero because $1 \otimes \beta^{\#} \mid{ }^{k} E_{2}^{*, 1}$ is injective. Since there are no higher differentials hitting ${ }^{k} E_{r}^{1, *}$ terms, $\left\{\left(1 \otimes \beta^{\#}\right) x\right\}$ is nonzero in ${ }^{k} E_{\infty}^{1,2}$. So $\beta^{\#} d_{2}^{0,1} M_{n}^{0 \#} \cong M_{n}^{0 \#}$ and is represented in ${ }^{k} E_{\infty}^{1,2}$. 
On the other hand, because the differential is a derivation, im $d_{2}^{1, *}$ is represented in

$$
\left({ }^{k} E_{\infty}^{1,0} \cup{ }^{k} E_{\infty}^{1,1}\right) \cup H^{*}\left(M_{n}^{0} ; \mathbf{Z} / p\right)={ }^{k} E_{\infty}^{2,1} \cup H^{*}\left(M_{n}^{0} ; \mathbf{Z} / p\right)
$$

or in a lower filtration. Therefore $\operatorname{im}\left(\beta^{\#} d_{2}^{0,1}\right) \cap \operatorname{im}\left(d_{2}^{1, *}\right)=0$.

We conclude that $d_{3}^{0,2} \mid \beta^{\#} M_{n}^{0 \#}$ is injective. Hence so is $d_{3}^{0, *} \mid S^{*}\left(\beta^{\#} M_{n}^{0 \#}\right)$.

5.4 Lemma. $H_{2}\left(\mathrm{SL}_{n} R_{k} ; M_{n}^{0}\right) \leqslant R \oplus R$ whenever $k \geqslant 2$.

Proof. In the spectral sequence

$$
{ }^{k} D_{* *}^{2}=H_{*}\left(\mathrm{SL}_{n} R ; H_{*}\left(G_{n}^{k} ; M_{n}^{0}\right)\right) \Rightarrow H_{*}\left(\mathrm{SL}_{n} R_{k} ; M_{n}^{0}\right), \quad{ }^{k} D_{1,1}^{2}=0
$$

(by 5.1(iii) and 3.1) and ${ }^{k} D_{2,0}^{2}=R$ (by 5.1(iv)). Further, there is a sequence

$$
\left(H_{2} G_{n}^{k} \otimes M_{n}^{0}\right)_{\mathrm{SL}_{n} R} \rightarrow^{k} D_{0,2}^{2}=\left(H_{2}\left(G_{n}^{k} ; \mathbf{Z} / p\right) \otimes M_{n}^{0}\right)_{\mathrm{SL}_{n} R} \rightarrow\left(\operatorname{Tor}\left(M_{n}^{0}, M_{n}^{0}\right)\right)_{\mathrm{SL}_{n} R} \text {, }
$$

where the final term is isomorphic to $\left(M_{n}^{0} \otimes M_{n}^{0}\right)_{\mathrm{SL}_{n} R}$.

Take $k>2$. Using the notation and proof of 3.4 , there are exact sequences

$$
U^{k} \otimes M_{n}^{0} \nrightarrow H_{2} G_{n}^{k} \otimes M_{n}^{0} \rightarrow J_{n} \otimes M_{n}^{0} ; \quad \tilde{U}^{k} \otimes M_{n}^{0} \nrightarrow U^{k} \otimes M_{n}^{0} \rightarrow M_{n}^{0} \otimes M_{n}^{0},
$$

where $\mathrm{SL}_{n} R$ acts trivally on $\tilde{U}_{k}$. Take $\mathrm{SL}_{n} R$-coinvariance of these and apply the A.2 results $\left(J_{n} \otimes M_{n}\right)_{\mathrm{SL}_{n} R}=0$ and $\left(M_{n}^{0} \otimes M_{n}^{0}\right)_{\mathrm{SL}_{n} R}=R$ to conclude that $\left(H_{2} G_{n}^{k} \otimes M_{n}^{0}\right)_{\mathrm{SL}_{n} R}$ is dominated by $R$. Thus ${ }^{k} D_{0,2}^{2} \leqslant R \otimes R$. When $k=2$, the same result holds because $H_{2} G_{n}^{2} \otimes M_{n}^{0} \cong \wedge^{2} M_{n}^{0} \otimes M_{n}^{0}$ and so by A.2(iv) has $\mathrm{SL}_{n} R$ coinvariance $R$.

The next lemma shows that there is a surjection im $d_{2,1}^{2} \rightarrow R$. Since

$$
H_{2}\left(\mathrm{SL}_{n} R_{k} ; M_{n}^{0}\right)={ }^{k} D_{2,0}^{2} \oplus{ }^{k} D_{1,1}^{2} \oplus{ }^{k} D_{0,2}^{3},
$$

it will prove this lemma.

5.5 LEMmA. In the spectral sequence ${ }^{k} D_{* *}^{2} \Rightarrow H_{*}\left(\mathrm{SL}_{n} R_{k} ; M_{n}^{0}\right)$ of 5.4 (proof), $\operatorname{im} d_{2,1}^{2}$ maps onto $R$.

Proof. The case $k=2$ is covered by [ALS, Theorem 2.2], which shows that $\operatorname{im} d_{2,1}^{2}$ maps onto $\left(\operatorname{Tor}\left(M_{n}^{0}, M_{n}^{0}\right)\right)_{\mathrm{SL}_{n} R}$.

If $k \geqslant 3$, let $\tau: M_{n}^{0}\left(t^{k}\right) \rightarrow M_{n}^{0}\left(t^{2}\right)$ be the coefficient isomorphism. The spectral sequence map $\pi_{*} \tau_{*}:{ }^{k} E_{0,2}^{2} \rightarrow{ }^{2} E_{0,2}^{2}$ induces an isomorphism between the terms $\left(\operatorname{Tor}\left(M_{n}^{0}, M_{n}^{0}\right)\right)_{\mathrm{SL}_{n} R} ;$ and $\pi_{*} \tau_{*}$ also induces an isomorphism

$$
H_{2}\left(\mathrm{SL}_{n} R ; H_{1}\left(G_{n}^{k} ; M_{n}^{0}\left(t^{k}\right)\right)\right) \rightarrow H_{2}\left(\mathrm{SL}_{n} R ; H_{1}\left(M_{n}^{0} ; M_{n}^{0}\left(t^{2}\right)\right)\right),
$$

because $\pi_{*}: H_{1}\left(G_{n}^{k} ; \mathbf{Z} / p\right) \cong H_{1}\left(G_{n}^{2} ; \mathbf{Z} / p\right)$ by 3.1(i). The lemma, and hence Lemma 5.4 , follows.

5.6 Proposition. If $k>2, \# \operatorname{ker}\left(\pi_{*}^{k}: H_{3} \mathrm{SL}_{n} R_{k} \rightarrow H_{3} \mathrm{SL}_{n} R_{k-1}\right) \leqslant \#(R)^{3}$.

Proof. The spectral sequence $(5.1)(k)$ has $E_{0,3}^{\infty}=0$ (by 5.3), $E_{1,2}^{\infty} \leqslant R$ (by 5.2) and $E_{2,1}^{\infty} \leqslant R \oplus R$ (by 5.4). 
We next investigate

$$
H_{3} \mathrm{SL}_{n} R_{k-1} / \pi_{*} H_{3} \mathrm{SL}_{n} R_{k}
$$

to estimate $\# H_{3} \mathrm{SL}_{n} R_{k} / \# H_{3} \mathrm{SL}_{n} R_{k-1}$.

5.7 Proposition. If $k>2$, there is an exact sequence

$$
\operatorname{ker} \pi_{*}^{k} \nrightarrow H_{3} \mathrm{SL}_{n} R_{k} \stackrel{\pi_{*}^{k}}{\rightarrow} H_{3} \mathrm{SL}_{n} R_{k-1} \rightarrow R .
$$

Hence with Proposition 5.6 we have

$$
\# \mathrm{H}_{3} \mathrm{SL}_{n} R_{k} / \# \mathrm{H}_{3} \mathrm{SL}_{n} R_{k-1} \leqslant(\# R)^{2} \text {. }
$$

Proof. In the spectral sequence $(5.1)(k), E_{0,2}^{2}=\left(\left(H_{2} M_{n}^{0}\left(t^{k}\right)\right)_{\mathrm{SL}_{n} R}\right)_{G_{n}^{k}}=0$ by 5.1(i). Therefore coker $\pi_{*}^{k}=\operatorname{im} d_{3,0}^{2}$, and this is $E_{1,1}^{2}$ because $H_{2} \operatorname{SL}_{n} R_{k} \equiv K_{2}\left(n, R_{k}\right)$ $=0[\mathrm{DS}, 4.4]$. Finally, $E_{1,1}^{2}=H_{1}\left(\mathrm{SL}_{n} R ; M_{n}^{0}\left(t^{k}\right)\right) \oplus\left(H_{1} G_{n}^{k} \otimes M_{n}^{0}\right)_{\mathrm{SL}_{n} R} \cong R$, following the proof of 5.2 and substituting the isomorphism of 3.1(i) and the results 5.1(i) and A.2(i).

The main theorem is an easy corollary to 5.7 and the following theorem.

5.8 Theorem (VAN DER KAllen AND StienSTRA). If $q \gg k$, there is an isomorphism

$$
\Delta_{k}: K_{1}\left(R_{2 k},(t)\right) /\left\{1-\alpha t^{k}: \alpha \in R\right\} \rightarrow K_{2}\left(R_{q},\left(t^{k}\right)\right) .
$$

Proof. This is a special case of [VKS, 4.3], given that $K_{2} R_{q}=0$ by the previously quoted result of Dennis and Stein.

Note that because $K_{2} R_{k}=0, K_{3}\left(R_{k},(t)\right)=H_{3} \mathrm{SL} R_{k} / H_{3} \mathrm{SL} R$.

5.9 THEOREM. If $R$ is a finite field of odd characteristic $p$, there is an isomorphism

$$
\partial^{-1} \circ \Delta_{k}: K_{1}\left(R_{2 k},(t)\right) /\left\{1-\alpha t^{k}: \alpha \in R\right\} \rightarrow K_{3}\left(R_{k},(t)\right),
$$

where $\Delta_{k}$ is as in 5.8 and $\partial: K_{3}\left(R_{k},(t)\right) \rightarrow K_{2}\left(R_{q},\left(t^{k}\right)\right)$ is the connecting homomorphism in the long exact $K$-sequence $(q \gg k)$.

If $n$ is large then also $H_{3} \mathrm{SL}_{n} R_{k} / H_{3} \mathrm{SL}_{n} R \cong K_{3}\left(R_{k},(t)\right)$ under the map induced by the inclusion $\mathrm{SL}_{n} R_{k} \rightarrow \mathrm{SL} R_{k}$.

Proof. If $k=2$ or 3 the theorem comes from [ALS, Theorem 1.1], given the stability of the generators used in that proof. Inductive application of (5.6) yields the group order estimate

$$
\# \mathrm{H}_{3} \mathrm{SL}_{n} R_{k} / \mathrm{H}_{3} \mathrm{SL}_{n} R \leqslant(\# R)^{2 k-2} \text {. }
$$

However, the order of the domain of $\partial^{-1} \circ \Delta_{k}$ is $(\# R)^{2 k-2}$; since $\partial$ is onto, $\# H_{3} \mathrm{SL} R_{k} / H_{3} \mathrm{SL} R$ must be at least as great. This implies the theorem when $n=\infty$. The general case comes from the stability theorems of, for example, [VK].

Appendix. This Appendix investigates the $\mathrm{SL}_{n} R$-structure of various modules related to $M_{n}^{0 \otimes i}, i=2$ or 3 . There are 2 subsections.

Notation is as in $\S 2$. 
A.1 Lemma. Let $I$ be the $\mathrm{SL}_{n} R$-submodule of $M_{n}^{0} \otimes M_{n}^{0}$ generated by $\underline{1}_{12} \otimes \underline{1}_{23}+$ $\underline{1}_{43} \otimes \underline{1}_{14}$. Let $\left(M_{n}^{0} \otimes M_{n}^{0}\right)^{0}$ be the kernel of the quotient map of $M_{n}^{0} \otimes M_{n}^{0}$ onto its $\mathrm{SL}_{n} R$-coinvariance $R[\mathbf{K 3}, 3.7]$. Let $\operatorname{St}(R, R)$ be the additive Steinberg group (defined in $[\mathbf{K 4}, 1.4])$ where by $[\mathbf{K 4}, 2.15]$ there is an epimorphism $\phi: \operatorname{St}(R, R) \rightarrow M_{n}^{0}$ such that $(\operatorname{St}(R, R), \phi)$ is the universal $\mathrm{SL}_{n} R$-central extension of $M_{n}^{0}$, with kernel $\Omega$.

Then

(i) $(I)_{\mathrm{SL}_{n} R}=0$.

(ii) $\left(M_{n}^{0^{n}} \otimes M_{n}^{0}\right)^{0} / I \cong \operatorname{St}(R, R)$, and if $D^{0}:\left(M_{n}^{0} \otimes M_{n}^{0}\right)^{0} / I \rightarrow M_{n}^{0}$ is the map induced by the Lie bracket, $\left(\left(M_{n}^{0} \otimes M_{n}^{0}\right)^{0} / I, D^{0}\right)$ is the universal $\mathrm{SL}_{n} R$-central extension of $M_{n}^{0}$.

(iii) $\left(M_{n}^{0} \otimes M_{n}^{0}\right) / I \cong \operatorname{St}(R, R) \oplus R$.

Proof. (i) $I$ is $\mathrm{SL}_{n} R$-generated by $\left(e_{15}-1\right) \cdot\left(\underline{1}_{52} \otimes \underline{1}_{23}+\underline{1}_{43} \otimes \underline{1}_{54}\right)$ (use the actions given in 2.3).

(ii) We will use the following definitions:

$\alpha, \beta, \gamma, \varepsilon$ and $\mu$ are arbitrary elements in $R ;$

$D:\left(M_{n}^{0} \otimes M_{n}^{0}\right) / I \rightarrow M_{n}^{0}$ is the map induced by $[$,$] ;$

$$
F_{i j}(\alpha, \beta)=\underline{\alpha}_{i j} \otimes \underline{\beta}_{j i} \in M_{n}^{0} \otimes M_{n}^{0}
$$

$\Psi: R \otimes R \rightarrow\left(M_{n}^{0} \otimes M_{n}^{0}\right) / I$ is: $\alpha \otimes \beta \rightarrow\left\{F_{12}(\alpha, \beta)+F_{23}(\alpha, \beta)-F_{13}(\alpha, \beta)\right\}$.

The proof is divided into two parts. The first shows that $\Psi$ is equivariant and that $\operatorname{im} \Psi=\operatorname{ker} D$, so that $\left(\left(M_{n}^{0} \otimes M_{n}^{0}\right)^{0} / I, D^{0}\right)$ is $\mathrm{SL}_{n} R$-central; the second shows that it is universal by exhibiting a map $\sigma:\left(M_{n}^{0} \otimes M_{n}^{0}\right)^{0} / I \rightarrow \operatorname{St}(R, R)$ with $\phi \circ \sigma=D^{0}$.

A. Define a group homomorphism $g: M_{n}^{0} \rightarrow\left(\left(M_{n}^{0} \otimes M_{n}^{0}\right) / I\right) / \operatorname{im} \Psi$ by choosing for each pair $i \neq j$, an $m \notin\{i, j\}$ and setting $g\left(\underline{\alpha}_{i j}\right)=\left\{\underline{1}_{i m} \otimes \underline{\alpha}_{m j}\right\}$, and, if $j>1$, $g\left(\underline{\underline{\alpha}}_{1 j}\right)=\left\{\underline{1}_{j 1} \otimes \underline{\alpha}_{1 j}\right\}$. We are going to show that $g$ is an epimorphism. Use 2.3 to check that im $\Psi$ lies in ker $D$; hence [, ] induces an epimorphism

$$
\left(\left(M_{n}^{0} \otimes M_{n}^{0}\right) / I\right) / \mathrm{im} \Psi \rightarrow M_{n}^{0}
$$

which is a left inverse to $g$, so that $\operatorname{im} \Psi=\operatorname{ker} D$ as claimed.

Consider each of the ten elements listed below as representative of the subset of the canonical Z-basis of $M_{n}^{0} \otimes M_{n}^{0}$ which can be obtained from (a) the action of the permutation matrices and (b) switching of the modules $M_{n}^{0}$ which form the tensor product. (Recall that $\underline{\ddot{\alpha}}_{i j}=\underline{\ddot{\alpha}}_{i m}+\underline{\ddot{\alpha}}_{m j}=-\underline{\ddot{\alpha}}_{j i}, m \notin\{i, j\}$.) The subsets are based on subscript configurations, and partition the basis. It thus suffices to show that the projections of these subsets to $\left(\left(M_{n}^{0} \otimes M_{n}^{0}\right) / I\right) / \mathrm{im} \Psi$ lie in the image of $g$.
1. $\underline{\alpha}_{12} \otimes \underline{\beta}_{34}$
2. $\ddot{\ddot{\alpha}}_{12} \otimes \underline{\beta}_{34}$
3. $\underline{\alpha}_{12} \otimes \underline{\beta}_{32}$
5. $\underline{\alpha}_{12} \otimes \underline{\beta}_{12}$
6. $\underline{\alpha}_{12} \otimes \underline{\beta}_{23}$
7. $\underline{\alpha}_{12} \otimes \underline{\beta}_{13}$
4. $\underline{\alpha}_{12} \otimes \underline{\beta}_{13}$
9. $\quad \underline{\alpha} \otimes \ddot{\beta}_{13}$
10. $\underline{\alpha}_{12} \otimes \underline{\beta}_{21}$.
8. $\quad \underline{\alpha}_{12} \otimes \underline{\beta}_{23}$

All elements of type 1-5 are zero modulo $I$. Look at $\left(e_{m 1}(\alpha)-1\right) \cdot \underline{1}_{12} \otimes \underline{1}_{34}$, $\left(e_{m 3}(\beta)-1\right) \cdot \underline{\alpha}_{12} \otimes \underline{1}_{34},\left(e_{12}-1\right) \cdot \underline{\alpha}_{21} \otimes \underline{\beta}_{34}$, etc., and use the symmetry of the $\mathrm{SL}_{n} R$-action on the modules $M_{n}^{0}$ forming the tensor product, plus the action of the 
permutation matrices. I also contains elements of the types $\underline{\gamma \alpha}_{12} \otimes \underline{\beta}_{23}-\underline{\gamma}_{14} \otimes \alpha \underline{\beta}_{43}$ and $\gamma \alpha_{23} \otimes \ddot{\beta}_{12}-\underline{\alpha}_{43} \otimes \gamma \beta_{24}$-consider $\left(e_{42}(-\alpha)-1\right) \cdot \underline{\gamma}_{14} \otimes \underline{\beta}_{23}$, etc. Similarly, it contains elements of type

$$
\text { (A.1) } \quad \underline{\gamma \alpha}_{12} \otimes \underline{\beta}_{23}-\underline{\alpha}_{14} \otimes \underline{\gamma \beta}_{42}\left(\left(e_{42}(-\gamma)-1\right) \cdot \underline{\alpha}_{14} \otimes \underline{\beta}_{23}+\underline{\gamma \alpha}_{12} \otimes \underline{\gamma \beta}_{42}\right) \text {. }
$$

The class of each element of type 6-8 is in im $g$. As above, $\underline{\varepsilon}_{i m} \otimes \underline{\mu}_{m j} \equiv \underline{\ddot{\alpha}}_{i m} \otimes \underline{\beta}_{i j}$ $\equiv \underline{\alpha}_{i j} \otimes \underline{\beta}_{j m}$ modulo $I$, whenever $\alpha \beta=\varepsilon \mu$ and $i, j$ and $m$ are distinct. The same sort of reasoning used above, applied to the generator $\underline{1}_{13} \otimes \underline{1}_{32}+\underline{1}_{42} \otimes \underline{1}_{14}$, shows that modulo $I,-\underline{1}_{i m} \otimes \alpha \underline{\beta}_{m j} \equiv \underline{\alpha}_{s j} \otimes \underline{\beta}_{i s} \equiv \underline{\ddot{\alpha}}_{j s} \otimes \underline{\beta}_{i j} \equiv \underline{\alpha}_{i j} \otimes \underline{\beta}_{i s}$ whenever $s, m, i$ and $j$ are mutually distinct.

We can now check that $\Psi$ is an equivariant map; e.g. if $m \notin\{1,2,3\}$,

$$
\left(e_{i m}(-\gamma)-1\right) \cdot \Psi(\alpha, \beta)=\underline{\alpha}_{12} \otimes \underline{\gamma \beta}_{2 m}-\underline{\alpha}_{13} \otimes \underline{\beta \gamma}_{3 m} \equiv 0 \bmod I
$$

and

$$
\begin{gathered}
\left(e_{12}(-\gamma)-1\right) \cdot \Psi(\alpha, \beta)=\underline{\alpha}_{12} \otimes\left(\underline{\beta} \underline{\ddot{\gamma}}_{21}-\underline{\gamma}_{12}^{2}\right) \\
-\underline{\alpha \gamma}_{13} \otimes \underline{\beta}_{32}-\underline{\alpha}_{13} \otimes \underline{\beta \gamma}_{32} \equiv 0 \bmod I .
\end{gathered}
$$

The class of each type 10 element is in im $g$. Take $i \neq j,\{i, j\} \cap\{a, b\}=\varnothing$ and $\alpha \beta=\varepsilon \mu$. Look at $\left(e_{21}-1\right)\left(\underline{\alpha} \underline{\gamma}_{13} \otimes \underline{\beta}_{32}-\underline{\alpha}_{14} \otimes \underline{\gamma}_{42}\right)$ and permutations to see that

$$
-F_{i a}(\alpha \gamma, \beta)+F_{j a}(\alpha \gamma, \beta)+F_{i b}(\alpha, \gamma \beta)-F_{j b}(\alpha, \gamma \beta) \in I \text {. }
$$

Apply $\left(e_{j i}-1\right)$ to $\underline{\varepsilon}_{i a} \otimes \underline{\mu}_{a j}+\underline{\alpha}_{b j} \otimes \underline{\beta}_{i b}$ to see that

$$
-F_{j a}(\varepsilon, \mu)+F_{i a}(\varepsilon, \mu)+F_{b i}(\alpha, \beta)-F_{b j}(\alpha, \beta) \in I \text {. }
$$

Hence modulo $I+\operatorname{im} \Psi$,

$$
F_{12}(\varepsilon, \mu)+F_{b 1}(\alpha, \beta)-F_{b 2}(\alpha, \beta) \equiv 0 ;
$$

i.e., $F_{b 2}(\alpha, \beta) \equiv F_{12}(\varepsilon, \mu)+F_{b 1}(\alpha, \beta) \equiv F_{j 2}(\varepsilon, \mu)+F_{b j}(\alpha, \beta)$, by (A.3) so $F_{b j}(\alpha, \beta)$ $\equiv F_{b 2}(\alpha, \beta)-F_{j 2}(\alpha, \beta) \equiv F_{b a}(1, \alpha \beta)-F_{j a}(1, \alpha \beta)$, by (A.2). Set $a=1$.

The class of each type 9 element is in im $g$. As shown above $\underline{\alpha}_{12} \otimes \underline{\beta}_{13}-\underline{\alpha}_{14} \otimes \underline{\beta}_{43}$ $\in I$. Apply $\left(e_{31}-1\right)$ to it and use the facts that $F_{31}(\alpha, \beta)+F_{14}(\alpha, \beta)-F_{34}(\alpha, \beta)$ $\in(I+\operatorname{im} \Psi)$, and, modulo $I,-\underline{\ddot{\alpha}}_{12} \otimes \underline{\beta}_{31} \equiv \underline{\alpha}_{34} \otimes \underline{\beta}_{41}$. This gives $\underline{\ddot{\alpha}}_{12} \otimes \underline{\beta}_{13} \equiv-\underline{\alpha}_{31}$ $\otimes \beta_{31} \equiv 0$ in $\left(\left(M_{n}^{0} \otimes M_{n}^{0}\right) / I\right) /$ im $\Psi$.

This completes part A.

B. Before going into the main part of the proof, we recall some facts about Hochschild homology [I].

If $S$ is any associative ring and $N$ is an $S$-bimodule, the Hochschild homology groups $H H_{*}(S, N)$ are defined as the homology of the complex $C_{H}(S, N)=\left(\left(S^{\otimes *}\right)\right.$ $\left.\otimes N, \delta_{H}\right)$, where

$$
\begin{aligned}
\delta_{H}\left(s_{1} \otimes \cdots \otimes s_{m} \otimes x\right)= & s_{1} \otimes \cdots \otimes s_{m-1} \otimes s_{m} x \\
& +\sum_{i=1}^{m-1}(-1)^{i} s_{1} \otimes \cdots \otimes s_{i} s_{i+1} \otimes \cdots \otimes x \\
& +(-1)^{m} s_{2} \otimes \cdots \otimes s_{n} \otimes x s_{1} .
\end{aligned}
$$


The Morita equivalence $H H_{*}\left(M_{n}^{0}, M_{n}^{0}\right) \rightarrow H H_{*}(R, R)$ is induced by the chain equivalence

$$
\begin{array}{r}
\operatorname{Tr}\left(X_{1} \otimes \cdots \otimes X_{m}\right)=\sum_{a, b, \ldots, z=1}^{n}\left(x_{a b}^{1} \otimes x_{b c}^{2} \otimes \cdots \otimes x_{y z}^{m-1} \otimes x_{z a}^{m}\right) \\
\quad \text { where } X_{i}=\sum_{a, b=1}^{n} \underline{x}_{a b}^{i} a b .
\end{array}
$$

Define $f: \quad M_{n}^{0} \otimes M_{n}^{0} \rightarrow H H_{1}(R, R)=\Omega$ by $f(a \otimes b)=\{\operatorname{Tr}(a \otimes b)\} . \quad$ In $C_{H}\left(M_{n}^{0}, M_{n}^{0}\right)$, for any $r \in \mathrm{SL}_{n} R$ and $a, b \in M_{n}^{0}$, compute

$$
r a r^{-1} \otimes r b r^{-1}=r \otimes[a, b] r^{-1}+a \otimes b+\delta_{H}\left(r a r^{-1} \otimes r \otimes b r^{-1}-r \otimes a \otimes b r^{-1}\right) .
$$

Thus, since $\operatorname{Tr}$ is a chain equivalence,

$$
f\left(r a r^{-1} \otimes r b r^{-1}\right)=f\left(r \otimes[a, b] r^{-1}\right)+f(a \otimes b) .
$$

As a group, $\operatorname{St}(R, R) \cong \Omega \oplus M_{n}^{0}$. Define $\sigma:\left(M_{n}^{0} \otimes M_{n}^{0}\right)^{0} \rightarrow \operatorname{St}(R, R)$ by

$$
\sigma(a \otimes b)=(f(a \otimes b),[a, b]) \text {. }
$$

Check that $\sigma\left(\underline{1}_{12} \otimes \underline{1}_{23}+\underline{1}_{43} \otimes \underline{1}_{14}\right)=0$. Hence if we can show that $\sigma$ is an $\mathrm{SL}_{n} R$-module map, we will have an induced map

$$
\left(M_{n}^{0} \otimes M_{n}^{0}\right)^{0} / I \rightarrow \operatorname{St}(R, R)
$$

which is inverse to the canonical map.

With the identification $\operatorname{St}(R, R)=\Omega \oplus M_{n}^{0}$, the $\mathrm{SL}_{n} R$-action is given by

$$
r \cdot(h, u)=\left(f\left(r \otimes u r^{-1}\right)+h, r u r^{-1}\right) ; \quad r \in \mathrm{SL}_{n} R, h \in \Omega, u \in M_{n}^{0}
$$

(to check this, one first checks that the action is well defined, then that it is compatible with the usual description of the action on $\operatorname{St}(R, R)$ defined in terms of the generators $\left.y_{i j}(a)[\mathbf{K 4}, 1.4]\right)$. Then because $\mathrm{SL}_{n} R$ acts on $M_{n}^{0}$ by conjugation,

$$
\begin{gathered}
\sigma(r \cdot(a \otimes b))=\sigma\left(r a r^{-1} \otimes r b r^{-1}\right)=\left(f\left(r a r^{-1} \otimes r b r^{-1}\right), r[a, b] r^{-1}\right) \\
\stackrel{(\text { A.4) }}{=}\left(f \otimes[a, b] r^{-1}+f(a \otimes b), r[a, b] r^{-1}\right) \\
=r \cdot(f(a \otimes b),[a, b])=r \cdot \sigma(a \otimes b) .
\end{gathered}
$$

Thus $\sigma$ is an $\mathrm{SL}_{n} R$-module map.

(iii) This is read from the commutative diagram:

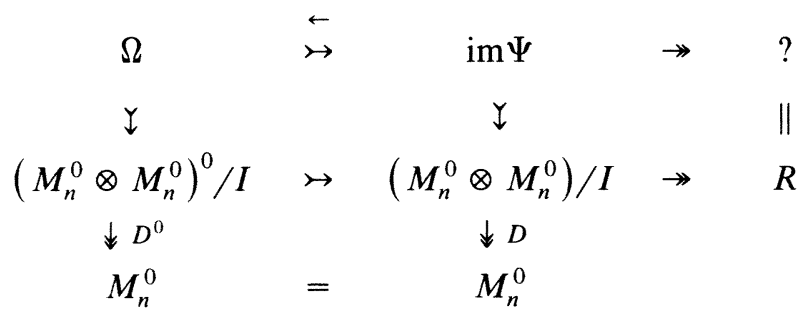

A.2 LEMMA. (i) $\left(M_{n}^{0} \otimes M_{n}^{0}\right)_{\mathrm{SL}_{n} R}=R$, with $\alpha \leftrightarrow\left\{\underline{1}_{12} \otimes \underline{\alpha}_{21}\right\}$,

(ii) $\left(\wedge^{2} M_{n}^{0}\right)_{\mathrm{SL}_{n} R}=R / 2 R$, and $\left(S^{2} M_{n}^{0}\right)_{\mathrm{SL}_{n} R}=R$, 
(iii) $\left(\left(M_{n}^{0}\right)^{\otimes 3}\right)_{\mathrm{SL}_{n} R}=R \oplus R$ with $(\alpha, \beta) \leftrightarrow\left\{\underline{1}_{12} \otimes \underline{1}_{23} \otimes \underline{\alpha}_{31}\right\}+\left\{\underline{1}_{23} \otimes \underline{1}_{12} \otimes\right.$ $\left.\beta_{31}\right\}$,

(iv) $\left(\bigwedge^{2} M_{n}^{0} \otimes M_{n}^{0}\right)_{\mathrm{SL}_{n} R}=R$, with $\alpha \leftrightarrow\left\{\underline{1}_{12} \underline{1}_{23} \otimes \underline{\alpha}_{31}\right\}$,

(v) $\left(\wedge^{3} M_{n}^{0}\right)_{\mathrm{SL}_{n} R}=R$, with $\alpha \leftrightarrow\left\{\underline{1}_{12} \underline{1}_{23} \underline{\alpha}_{31}\right\}$,

(vi) $\left(J_{n} \otimes M_{n}^{0}\right)_{\mathrm{SL}_{n} R}=0$.

Proof. (i) (Kassel $[\mathbf{K 3}, 3.7]$ ) The coinvariance is detected by the equivariant map

$T: M_{n}^{0} \otimes M_{n}^{0} \rightarrow R, \quad$ where $T(a \otimes b)=\operatorname{Tr}(a b)$.

(ii) $\wedge^{2} M_{n}^{0}$ is $M_{n}^{0} \otimes M_{n}^{0}$ quotiented by the group generated by $\{a \otimes b+b \otimes a$, $\left.a \otimes a ; a, b \in M_{n}^{0}\right\}$, so the map induced by $T$ detects the coinvariance [K3, 3.7]. Similarly $S^{2} M_{n}^{0}$ is the quotient of $M_{n}^{0} \otimes M_{n}^{0}$ by the group generated by $\{a \otimes b-b$ $\left.\otimes a ; a, b \in M_{n}^{0}\right\}$; again, the map induced by $T$ detects.

(iii) Let $I$ be the $\mathrm{SL}_{n} R$-submodule of $M_{n}^{0} \otimes M_{n}^{0}$ generated by $\underline{1}_{12} \otimes \underline{1}_{23}+\underline{1}_{42} \otimes$ $\underline{1}_{14}$. Lemma A.1(ii) exhibits a sequence $\operatorname{im} \Psi \nrightarrow\left(M_{n}^{0} \otimes M_{n}^{0}\right) / I \stackrel{D}{\rightarrow} M_{n}^{0}$, where $D$ is induced by the commutator map [, ] of 3.2, and $\mathrm{SL}_{n} R$ acts trivially on im $\Psi$. Since $\left(M_{n}^{0}\right)_{\mathrm{SL}_{n} R}=0$,

$$
D_{*}:\left(\left(\left(M_{n}^{0} \otimes M_{n}^{0}\right) / I\right) \otimes M_{n}^{0}\right)_{\mathrm{SL}_{n} R} \rightarrow\left(M_{n}^{0} \otimes M_{n}^{0}\right)_{\mathrm{SL}_{n} R}=R
$$

is an isomorphism. We will compute $\left(I \otimes M_{n}^{0}\right)_{\mathrm{SL}_{n} R}$ by determining the $\mathrm{SL}_{n} R$ covariance classes of its generators, $\left\{\dot{y}_{a b}(\alpha)=\left(\underline{1}_{12} \otimes \underline{1}_{23}+\underline{1}_{43} \otimes \underline{1}_{14}\right) \otimes \underline{\dot{\alpha}}_{a b}: a \neq\right.$ $b\}$, where $\underline{\dot{\alpha}}_{i j}$ denotes "either $\underline{\alpha}_{i j}$ or $\underline{\alpha}_{i j}$ " and $\dot{y}_{i j}$ denotes "either $y_{i j}$ or $\ddot{y}_{i j}$ ". Congruence in what follows is with respect to coinvariance class.

If $a \notin\{2,3,4\}$, choose $c \notin\{1,2,4, a\}$. Then $y_{a b}(\alpha)=\left(e_{a c}-1\right) \cdot y_{c b}(\alpha)$ (or, if $c=b,\left(e_{a c}-1\right) \cdot \ddot{y}_{c 2}(\alpha)$, say). Apply this sort of argument again, to conclude

$$
\text { if } a \notin\{2,3,4\} \text { or } b \notin\{1,2,4\}, \quad y_{a b}(\alpha) \equiv 0 .
$$

If $a \notin\{2,3,4\}$ and $b \notin\{1,2,4\}, \quad \ddot{y}_{a b}(\alpha)=\left(e_{a b}-1\right) \cdot y_{b a}(\alpha)+y_{a b}(\alpha)$. This together with the identities $\ddot{y}_{a b}(\alpha)=\ddot{y}_{a 5}(\alpha)+\ddot{y}_{5 b}(\alpha)$ and $\ddot{y}_{a 5}(\alpha)=\ddot{y}_{5 a}(-\alpha)$, implies

$$
\ddot{y}_{a b}(\alpha) \equiv 0 \text { unless }\{a, b\} \cap\{2,4\} \neq \varnothing .
$$

Hence we need only consider $\{a, b\} \subset\{1,2,3,4\}$.

$P(i, j)$ is the permutation matrix $e_{i j} e_{j i}(-1) e_{i j}$. Check that if $1 \notin\{a, b\}, \dot{y}_{a b}=$ $\left(e_{15}-1\right) \cdot\left(P(5,1) \cdot \dot{y}_{a b}\right)$, and if $3 \notin\{a, b\},-y_{a b}=\left(e_{53}-1\right) \cdot\left(P(5,3) \cdot \dot{y}_{a b}\right)$.

This eliminates classes of all the generating set of $I \otimes M_{n}^{0}$ other than $\left\{y_{31}(\alpha)\right\}$. However, the equivariant function on $M_{n}^{\otimes 3},: A \otimes B \otimes C \rightarrow \operatorname{Tr}(A B C)$, takes $y_{31}(\alpha)$ to $\alpha$, so there is a copy of $R$ in $\left(I \otimes M_{n}^{0}\right)_{\mathrm{SL}_{n} R}$. Thus there is a split sequence

$$
R=\left(I \otimes M_{n}^{0}\right)_{\mathrm{SL}_{n} R} \stackrel{\iota}{\rightarrow}\left(M_{n}^{0 \otimes 3}\right)_{\mathrm{SL}_{n} R} \stackrel{D_{*}}{\rightarrow} R,
$$

where $\iota(\alpha)$ is the class of $\underline{1}_{12} \otimes \underline{1}_{23} \otimes \underline{\alpha}_{31}+\underline{1}_{43} \otimes \underline{1}_{14} \otimes \underline{\alpha}_{31}$, and $D_{*}^{-1}(\alpha)$ is the class of $\underline{1}_{12} \otimes \underline{1}_{23} \otimes \underline{\alpha}_{31}$.

(iv) The homology product $M_{n}^{0 \otimes 3} \rightarrow \wedge^{2} M_{n}^{0} \otimes M_{n}$ takes $y_{31}(\alpha)$ to

$$
(\cap \otimes 1) y_{31}(\alpha)=\underline{1}_{12} \underline{1}_{23} \otimes \underline{\alpha}_{31}-\underline{1}_{14} \underline{1}_{43} \otimes \underline{\alpha}_{31} \equiv-\left(e_{42}-1\right) \cdot\left(\underline{1}_{14} \underline{1}_{23} \otimes \underline{\alpha}_{31}\right) .
$$

$D_{*}^{-1}(\alpha)$ maps through $\underline{1}_{12} \underline{1}_{23} \otimes \underline{\alpha}_{31} \in \Lambda^{2} M_{n}^{0} \otimes M_{n}^{0}$ to $\underline{1}_{13} \otimes \underline{\alpha}_{31}$ in $M_{n}^{0} \otimes M_{n}^{0}$. Hence $\left(\wedge^{2} M_{n}^{0} \otimes M_{n}^{0}\right)_{\mathrm{SL}_{n} R}$ is as described. 
(v) By (iv), the homology product induces an epimorphism $R \rightarrow\left(\bigwedge^{3} M_{n}^{0}\right)_{\mathrm{SL}_{n} R}$. Observe this is an isomorphism, since there is an $\mathrm{SL}_{n} R$-invariant function in $\left(\wedge^{3} M_{n}^{0}\right)^{\#}, A \cap B \cap C \rightarrow \operatorname{Tr}(A B C-B A C), A, B, C \in M_{n}^{0}$, which takes $\underline{1}_{12} \cap \underline{1}_{23}$ $\cap \underline{\alpha}_{31}$ to $\alpha$.

(vi) $J_{n}$ is the image under the homology product of $L_{n}$ (see 3.2) whereas A.1(ii) proves $\left(L_{n} / I \equiv \operatorname{ker} D\right)$ is $\mathrm{SL}_{n} R$-invariant. Thus, if $I^{\prime}$ is the image of $I$ under the product, it suffices to show that $\left(I^{\prime} \otimes M_{n}^{0}\right)_{\mathrm{SL}_{n} R}=0$. But $\left(I^{\prime} \otimes M_{n}^{0}\right)_{\mathrm{SL}_{n} R}$ is generated by the class of $(\cap \otimes 1) y_{31}(\alpha)$, and this is the zero class in $\left(I^{\prime} \otimes M_{n}^{0}\right)_{\mathrm{SL}_{n} R}$ as well as in $\left(\bigwedge^{2} M_{n}^{0} \otimes M_{n}^{0}\right)_{\mathrm{SL}_{n} R}$.

\section{REFERENCES}

[A] J. Aisbett, K-groups of rings and the homology of their elementary matrix groups, J. Austral. Math. Soc. Ser. A 38 (1985), 268-274.

[ALSS] J. Aisbett, E. Lluis-Puebla, V. Snaith and C. Soulé, On $K_{*}(\mathbf{Z} / n)$ and $K_{*}\left(\mathbf{F}_{q}[t] /\left(t^{2}\right)\right)$, Mem. Amer. Math. Soc., Vol. 57, No. 329, 1985.

[ALS] J. Aisbett, E. Lluis-Puebla and V. Snaith, On $K_{3}$ of $\mathbf{F}_{q}[t] /\left(t^{2}\right)$ and $\mathbf{F}_{q}[t] /\left(t^{3}\right)$, J. Algebra (to appear).

[B] K. S. Brown, Cohomology of groups, Graduate Texts in Math., no. 87, Springer-Verlag, Berlin and New York, 1982.

[CE] H. Cartan and S. Eilenberg, Homological algebra, Princeton Univ. Press, Princeton, N. J., 1956.

[DS] K. Dennis and M. Stein, $K_{2}$ of discrete valuation rings, Adv. in Math. 18 (1975), 182-238.

[I] K. Igusa, What happens to Hatcher and Wagoner's formula for $\pi_{0} C(M)$ when the first Postnikov invariant of $M$ is nontrivial? Lecture Notes in Math., vol. 1046, Springer-Verlag, Berlin and New York, 1984, pp. 104-172.

[K1] C. Kassel, Un calcul d'homologie du groupe linéaire général, C. R. Acad. Sci. Paris 288 (1979), $481-483$.

[K2] _ Le groupe $K_{3}(\mathbf{Z}[\varepsilon]) n$ ' a pas de p-torsion pour $p \neq 2$ et 3, Lecture Notes in Math., vol. 966, Springer-Verlag, Berlin and New York, 1982, pp. 114-121.

[K3] _ K-théorie relative d'un idéal bilatèrie de carré nul, Lecture Notes in Math., vol. 854, Springer-Verlag, Berlin and New York, 1981, pp. 249-261.

[K4] Calcul algébrique de l'homologie de certains groups de matrices, J. Algebra 80 (1983), $235-260$.

[LS] E. Lluis-Puebla and V. Snaith, Determination of $K_{3}\left(\mathbf{F}_{p} l[t] /\left(t^{2}\right)\right)$ for primes $p \geqslant 5$, Current Trends in Algebraic Topology, CMS Conf. Proc., vol. 2.1, Amer. Math. Soc., Providence, R.I., 1984.

[L] J.-L. Loday, On the boundary map $K_{3}(\Lambda / I) \rightarrow K_{2}(\Lambda, I)$, Lecture Notes in Math., vol. 854, Springer-Verlag, Berlin and New York, 1981, pp. 262-268.

[M] S. Mac Lane, Homology, Springer-Verlag, Berlin, 1963.

[Ro] L. Roberts, $K_{2}$ of some truncated polynomial rings, Lecture Notes in Math., vol. 734, SpringerVerlag. Berlin and New York, 1980, pp. 249-278.

[S] J. Stienstra, On $K_{2}$ and $K_{3}$ of truncated polynomial rings, Lecture Notes in Math., vol. 854, Springer-Verlag, Berlin and New York, 1981, pp. 409-455.

[VK] W. van der Kallen, Homology stability for linear groups, Invent. Math. 60 (1980), 269-295.

[VKS] W. van der Kallen and J. Stienstra, The relative $K_{2}$ of truncated polynomial rings, J. Pure Appl. Algebra 34 (1984), 277-290.

\footnotetext{
Department of Mathematics, University of Queensland, St. Lucia, QueEnsland, Australia 4067
}

Current address: Electronics Research Laboratory, Defence Science and Technology Organisation, G.P.O. Box 2151, Adelaide 5001, Australia 